\title{
Dehn twists on nonorientable surfaces
}

\author{
by \\ Michał Stukow (Gdańsk)
}

\begin{abstract}
Let $t_{a}$ be the Dehn twist about a circle $a$ on an orientable surface. It is well known that for each circle $b$ and an integer $n, I\left(t_{a}^{n}(b), b\right)=|n| I(a, b)^{2}$, where $I(\cdot, \cdot)$ is the geometric intersection number. We prove a similar formula for circles on nonorientable surfaces. As a corollary we prove some algebraic properties of twists on nonorientable surfaces. We also prove that if $\mathcal{M}(N)$ is the mapping class group of a nonorientable surface $N$, then up to a finite number of exceptions, the centraliser of the subgroup of $\mathcal{M}(N)$ generated by the twists is equal to the centre of $\mathcal{M}(N)$ and is generated by twists about circles isotopic to boundary components of $N$.
\end{abstract}

1. Introduction. Let $N_{g, r}^{s}$ be a smooth, nonorientable, compact surface of genus $g$ with $r$ boundary components and $s$ punctures. If $r$ and/or $s$ is zero then we omit it from the notation. If we do not want to emphasise the numbers $g, r, s$, we simply write $N$ for a surface $N_{g, r}^{s}$. Recall that $N_{g}$ is a connected sum of $g$ projective planes and $N_{g, r}^{s}$ is obtained from $N_{g}$ by removing $r$ open disks and specifying a set $\Sigma$ of $s$ distinguished points in the interior of $N$.

Let $\mathcal{H}(N)$ be the group of all diffeomorphisms $h: N \rightarrow N$ such that $h$ is the identity on each boundary component and $h(\Sigma)=\Sigma$. By $\mathcal{M}(N)$ we denote the quotient group of $\mathcal{H}(N)$ by the subgroup consisting of the maps isotopic to the identity, where we assume that the isotopies fix $\Sigma$ and are the identity on each boundary component. $\mathcal{M}(N)$ is called the mapping class group of $N$. The mapping class group of an orientable surface is defined analogously, but we consider only orientation preserving maps. Usually we will use the same letter for a map and its isotopy class.

1.1. Background. In contrast to the mapping class groups of orientable surfaces, the nonorientable case has not been studied much. The first significant result is due to Lickorish [9], who proved that the mapping class

2000 Mathematics Subject Classification: Primary 57N05; Secondary 20F38, 57M99. Key words and phrases: mapping class groups, nonorientable surfaces, Dehn twists. Supported by KBN 1 P03A 02626. 
group of a closed nonorientable surface is generated by Dehn twists and a so-called crosscap slide (or a Y-homeomorphism). Later this generating set was simplified by Chillingworth [2], and extended to the case of punctured surfaces by Korkmaz [8]. Korkmaz also computed the first homology group of the mapping class groups of punctured nonorientable surfaces $[7,8]$. It is also known that the group $\mathcal{M}\left(N_{g}^{s}\right)$ is generated by involutions [11, 12].

At first glance it seems that it should be possible to derive some properties of $\mathcal{M}(N)$ from the properties of the mapping class group of its orientable double cover. Surprisingly, although it is known that $\mathcal{M}(N)$ is isomorphic to the centraliser of some involution in the mapping class group of the double cover of $N$ (see [1]), this idea has not led to any significant results.

One of the most fundamental properties of the mapping class group is that it acts on the set $\mathcal{C}$ of isotopy classes of circles. In the case of an orientable surface this observation leads to the most powerful tools in the study of mapping class groups.

For example the set $\mathcal{C}$ has simple structures of a simplicial complex, which lead to definitions of complexes of curves. This idea was the basic tool in finding a presentation of the mapping class group and also in obtaining some descriptions of its (co)homology groups (cf. [5] and references there).

Another example is the extension of the action of the mapping class group on $\mathcal{C}$ to the action on equivalence classes of measured foliations. This idea leads to the Thurston theory of surface diffeomorphisms (cf. [4]).

In either of these examples, it is of fundamental importance to understand the action of generators of $\mathcal{M}(N)$ on a single circle. Throughout this paper, we concentrate on a very basic result in this direction, namely on the well known formula for the intersection number

$$
I\left(t_{a}^{n}(b), b\right)=|n| I(a, b)^{2},
$$

which holds for any two circles $a$ and $b$ on an orientable surface and any integer $n$ (cf. Proposition 3.3 of [10]).

1.2. Main results. Our first result provides a formula for the action of a twist on a nonorientable surface, similar to (1.1) (cf. Theorem 3.3). To be more precise, we show that for generic two-sided circles $a$ and $b$ on $N$ such that $I(a, b)=|a \cap b|$, and any integer $n \neq 0$, we have

$$
I\left(t_{a}^{n}(b), b\right)=|n| I(a, b)^{2}-\sum_{i=1}^{u} k_{i}^{2},
$$

where $k_{1}, \ldots, k_{u}$ are nonnegative integers depending only on the mutual position of $a$ and $b$.

As an application of this result, we prove in Section 4 some algebraic properties of twists on nonorientable surfaces. Finally, in Section 6 we show that up to a finite number of exceptions, the centraliser of the subgroup 
generated by the twists is equal to the centre of $\mathcal{M}\left(N_{g, r}^{s}\right)$ and is generated by $r$ boundary twists (cf. Theorem 6.2). We end the paper with an appendix, which contains the description of two rather exceptional mapping class groups, namely those of a Klein bottle with one puncture and of a Klein bottle with one boundary component.

All the results presented are well known in the orientable case (cf. [6, 10]), but for nonorientable surfaces they are new. Moreover, we believe that the methods we develop will contribute to a further study of mapping class groups of nonorientable surfaces.

Since the strategy we follow is similar to that in [10], in some cases we omit detailed proofs referring the reader to the above article.

2. Preliminaries. By a circle on $N$ we mean an oriented simple closed curve on $N \backslash \Sigma$, which is disjoint from the boundary of $N$. Usually we identify a circle with its image. If $a_{1}$ and $a_{2}$ are isotopic, we write $a_{1} \simeq a_{2}$. If two circles $a$ and $b$ intersect, we always assume that they intersect transversely. According to whether a regular neighbourhood of a circle is an annulus or a Möbius strip, we call the circle two-sided or one-sided respectively. We say that a circle is essential if it does not bound a disk disjoint from $\Sigma$, and generic if it bounds neither a disk with fewer than two punctures nor a Möbius strip disjoint from $\Sigma$. Notice that the nonorientable surface $N_{g, r}^{s}$ admits a generic two-sided circle if and only if $N \neq N_{1}^{s}$ with $s \leq 2$ and $N \neq N_{1,1}$.

Following [10] we will say that circles $a$ and $b$ cobound $a$ bigon if there exists a disk whose boundary is the union of an arc of $a$ and an arc of $b$. Moreover, we assume that except the end points, these arcs are disjoint from $a \cap b$.

For any two circles $a$ and $b$ we define their geometric intersection number as follows:

$$
I(a, b)=\inf \left\{\left|a^{\prime} \cap b\right|: a^{\prime} \simeq a\right\} .
$$

In particular, if $a$ is a two-sided circle and $a \simeq b$ then $I(a, b)=0$.

The following proposition (cf. Proposition 3.2 of [10]) provides a very useful tool for checking if two circles are in a minimal position (with respect to $|a \cap b|)$.

Proposition 2.1. Let $a$ and $b$ be essential circles on $N$. Then $|a \cap b|=$ $I(a, b)$ if and only if $a$ and $b$ do not cobound a bigon.

Let $a$ be a two-sided circle. By definition, a regular neighbourhood of $a$ is an annulus $S_{a}$, so if we fix one of two possible orientations of $S_{a}$, we can define the Dehn twist $t_{a}$ about $a$ in the usual way. We emphasise that since we are dealing with nonorientable surfaces, there is no canonical way to choose the orientation of $S_{a}$. Therefore by a twist about $a$ we always 
mean one of two possible twists about $a$ (the second one is then its inverse). By a boundary twist we mean a twist about a circle isotopic to a boundary component. If $a$ is not generic then the Dehn twist $t_{a}$ is trivial. We will show that the converse is also true (cf. Corollary 4.5).

Other important examples of diffeomorphisms of a nonorientable surface are the crosscap slide and the puncture slide. They are defined as a slide of a crosscap and of a puncture, respectively, along a one-sided circle (for precise definitions and properties see [8]).

3. Action of a Dehn twist on a two-sided circle. For the rest of this section let us fix two-sided generic circles $a$ and $b$ such that $|a \cap b|=I(a, b)$.

3.1. Definitions. By a segment of $b$ (with respect to $a$ ) we mean any unoriented arc $p$ of $b$ satisfying $a \cap p=\partial p$. Similarly we define an oriented segment. If $p$ is an oriented segment, by $-p$ we mean the segment equal to $p$ as an unoriented segment but with reversed orientation, and by $|p|$ the unoriented segment determined by $p$. We call a segment $p$ of $b$ one-sided [two-sided] if the union of $p$ and an arc of $a$ connecting $\partial p$ is a one-sided [twosided] circle. An oriented segment is one-sided [two-sided] if the underlying unoriented segment is one-sided [two-sided].

Oriented segments $P P^{\prime}$ and $Q Q^{\prime}$ (not necessarily distinct) of $b$ are called adjacent if both are one-sided and there exists an open disk $\Delta$ on $N \backslash \Sigma$ with the following properties:

(1) $\partial \Delta$ consists of the segments $P P^{\prime}, Q Q^{\prime}$ of $b$ and the $\operatorname{arcs} P Q, P^{\prime} Q^{\prime}$ of $a$;

(2) $\Delta$ is disjoint from $a \cup b$ (see Figure 1).
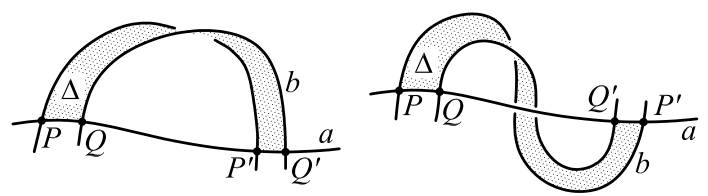

Fig. 1. Adjacent segments of $b$

REMARK 3.1. Let $p, q, p^{\prime}, q^{\prime}$ be oriented segments such that $p$ is adjacent to $q$ through a disk $\Delta$ and $p^{\prime}$ is adjacent to $q^{\prime}$ through $\Delta^{\prime}$. Then since $\Delta$ and $\Delta^{\prime}$ are disjoint from $a \cup b$, either $\Delta=\Delta^{\prime}$ or $\Delta \cap \Delta^{\prime}=\emptyset$. In particular if $\{p, q\} \neq\left\{p^{\prime}, q^{\prime}\right\}$ and $\{p, q\} \neq\left\{-p^{\prime},-q^{\prime}\right\}$ then $\Delta \cap \Delta^{\prime}=\emptyset$.

Oriented segments $p \neq q$ are called joinable if there exist oriented segments $p_{1}, \ldots, p_{k}$ such that $p_{1}=p, p_{k}=q$ and $p_{i}$ is adjacent to $p_{i+1}$ for $i=1, \ldots, k-1$. 
Unoriented segments are called adjacent [joinable] if they are adjacent [joinable] as oriented segments for some choice of orientations.

REMARK 3.2. Observe that if $p$ is a segment of $b$ then there are at most two segments of $b$ adjacent to $p$ (one on each side of $p$ ).

We now define a graph $\Gamma(a, b)$, which will help us to measure how much $I\left(t_{a}^{n}(b), b\right)$ differs from $|n| I(a, b)^{2}$ (cf. formula (1.1)). The vertices of $\Gamma(a, b)$ correspond to one-sided unoriented segments of $b$. If we have two segments which are adjacent through the disk $\Delta$, we join the vertices corresponding to these segments by an edge (labelled $\Delta$ ). So in particular, we do not exclude the possibility that there are multiple edges or loops.

Observe that segments $p \neq q$ are joinable if and only if the corresponding vertices of $\Gamma(a, b)$ can be connected by a path.

Having the above definitions, we can formulate the relationship between the action of a twist and the intersection number.

THEOREM 3.3. Let $a$ and $b$ be two-sided generic circles and let $k_{1}, \ldots, k_{u}$ be the numbers of vertices in the connected components of $\Gamma(a, b)$. Then for every integer $n \neq 0$,

$$
I\left(t_{a}^{n}(b), b\right)=|n| I(a, b)^{2}-\sum_{i=1}^{u} k_{i}^{2} .
$$

The rest of this section is devoted to the proof of the above theorem. The idea of the proof is very simple: construct the circle $t_{a}^{n}(b)$, perform all obvious reductions of $t_{a}^{n}(b) \cap b$ and count them, finally prove that there are no further reductions. However, the details of the proof are quite involved, and we first need some preparations.

3.2. Joinable segments. For two oriented joinable segments $p$ and $q$ define the distance between $p$ and $q$ to be the minimal $k$ such that there exist oriented segments $p_{1}, \ldots, p_{k}$ with $p_{1}=p, p_{k}=q$ and $p_{i}$ adjacent to $p_{i+1}$ for $i=1, \ldots, k-1$.

The following three lemmas, which contain the crucial properties of joinable segments, will be proved simultaneously.

Lemma 3.4. If $p$ is an oriented segment of $b$ then $p$ and $-p$ are not joinable.

LEMMA 3.5. Let $p$ and $q$ be oriented, joinable segments of $b$ at distance $k$, and let $p_{1}, \ldots, p_{k}$ be oriented segments such that $p_{1}=p, p_{k}=q$ and $p_{i}$ is adjacent to $p_{i+1}$ for $i=1, \ldots, k-1$. Then $\left|p_{i}\right| \neq\left|p_{j}\right|$ if $i \neq j$.

LEMma 3.6. Let $P_{1} P_{1}^{\prime}, \ldots, P_{k} P_{k}^{\prime}$ be oriented segments of $b$ such that $P_{i} P_{i}^{\prime}$ is adjacent to $P_{i+1} P_{i+1}^{\prime}$ through a disk $\Delta_{i}$ for $i=1, \ldots, k-1$. Moreover, assume that $P_{1} P_{1}^{\prime} \neq P_{k} P_{k}^{\prime}$ and the distance between these two segments is 
equal to $k$. Then $\Delta_{i} \cap \Delta_{j}=\emptyset$ for $i \neq j$, and the interior $\Delta$ of $\bigcup_{i=1}^{k-1} \bar{\Delta}_{i}$ is an open disk with the following properties:

(1) $\partial \Delta$ consists of the segments $P_{1} P_{1}^{\prime}, P_{k} P_{k}^{\prime}$ of $b$ and the arcs $P_{1} P_{k}, P_{1}^{\prime} P_{k}^{\prime}$

(2) $\stackrel{\text { of }}{\Delta} \cap$; $b=\left\{P_{1} P_{1}^{\prime}, \ldots, P_{k} P_{k}^{\prime}\right\}$;

(3) each of the sequences $P_{1}, \ldots, P_{k}$ and $P_{1}^{\prime}, \ldots, P_{k}^{\prime}$ is strictly monotone with respect to some orientation of a (cf. Figure 2).

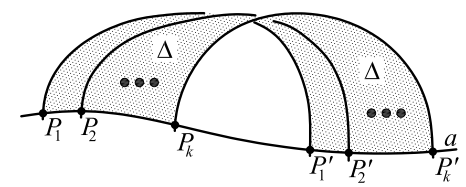

Fig. 2. Configuration of segments-Lemma 3.6

Proof of Lemmas 3.4-3.6. First observe that we have the implications:

(A) Lemma $3.4 \Rightarrow$ Lemma 3.5 ,

(B) Lemma $3.5 \Rightarrow$ Lemma 3.6.

In fact, in order to prove (A), let oriented segments $p_{1}, \ldots, p_{k}$ of $b$ be as in Lemma 3.5. Since $p_{1} \neq p_{k}$ (by the definition of joinability) and the sequence $p_{1}, \ldots, p_{k}$ is minimal with respect to $k$, we have $p_{i} \neq p_{j}$ for $i \neq j$. Moreover, by Lemma $3.4, p_{i} \neq-p_{j}$ for $i \neq j$.

To prove (B), observe that by Lemma 3.5, $\left|P_{i} P_{i}^{\prime}\right| \neq\left|P_{j} P_{j}^{\prime}\right|$ for $i \neq j$. Hence by Remark 3.1, $\Delta_{i} \cap \Delta_{j}=\emptyset$ for $i \neq j$ and one can think of $\Delta$ as the interior of a standard rectangle (obtained by gluing all $\bar{\Delta}_{i}$ 's along common boundary components) with two opposite sides glued to $a$. Now it is clear that $\Delta$ satisfies conditions (1)-(3) above.

Observe that the proofs of the above implications preserve distance, in the sense that if Lemma 3.4 is true for segments of distance $\leq k$ (i.e. $p$ and $-p$ are not joinable with distance $\leq k$ ), then Lemma 3.5 is also true for segments of distance $\leq k$. Similarly for implication (B).

The rest of the proof will be by induction (simultaneous for all three lemmas) on the distance between joinable segments.

Suppose first that $k=2$. We will prove Lemma 3.4; Lemmas 3.5 and 3.6 will follow by implications (A) and (B) above.

If $p$ is adjacent to $-p$ then there exists an open disk $\Delta$ with boundary consisting of $p,-p$ and two arcs of $a$ connecting $\partial p$. The best way to think about such a situation is that we have a rectangle (corresponding to $\Delta$ ) with two opposite sides glued by an orientation reversing map (these sides correspond to $p$ and $-p$ ). What we get is a Möbius strip with $a$ as the boundary circle, which is a contradiction, since $a$ is generic. 
Let $k \geq 3$, and assume that Lemmas 3.4-3.6 are true for joinable segments of distance less than $k$. By implications (A) and (B) it is enough to show that $p$ and $-p$ are not joinable with distance $k$.

Suppose that oriented segments $p_{1}, \ldots, p_{k}$ of $b$ are such that $p_{i}$ is adjacent to $p_{i+1}$ for $i=1, \ldots, k-1, p_{k}=-p_{1}$ and the distance between $p_{1}$ and $-p_{1}$ is equal to $k$. If $p_{k-1}=p_{1}$ then $p_{1}$ and $-p_{1}$ would have distance 2 , contrary to $k \geq 3$. Hence $p_{k-1} \neq p_{1}$ and we can apply Lemma 3.6 to the segments $p_{1}$ and $p_{k-1}$. Let $\Delta_{1}$ be an open disk provided by that lemma and let $\Delta_{2}$ be a disk given by adjacency of $p_{k-1}$ and $p_{k}=-p_{1}$. By Lemma 3.5, $\left|p_{i}\right| \neq\left|p_{j}\right|$ for $i \neq j, i, j \in 1, \ldots, k-1$, hence if we assume that $\Delta_{1} \cap \Delta_{2} \neq \emptyset$, then Remark 3.1 and the construction of $\Delta_{1}$ implies that $\Delta_{2}$ is a disk given by adjacency of $p_{k-2}$ and $p_{k-1}$ (this is because this is the only disk composing $\Delta_{1}$ which has $p_{k-1}$ as a boundary component). But this is impossible since $p_{k-2} \neq-p_{1}$ (otherwise the distance between $p_{1}$ and $-p_{1}$ would be less than $k$ ). Therefore $\Delta_{1} \cap \Delta_{2}=\emptyset$ and we claim that $\Delta=\bar{\Delta}_{1} \cup \bar{\Delta}_{2}$ is a Möbius strip with boundary equal to $a$, which leads to a contradiction, since $a$ is generic. In fact, $\Delta$ is obtained from a rectangle (corresponding to $\Delta_{1} \cup p_{k-1} \cup \Delta_{2}$ ) by identifying its two opposite sides (corresponding to $p_{1}$ and $-p_{1}$ ) by an orientation reversing map and then gluing the remaining side to $a$.

Since $a$ is two-sided, we have the notion of being on the same side of $a$ for germs of transversal arcs starting at the points of $a$. In particular, if $P$ is an end point of a segment $p$ and $Q$ of $q$ then by $P$ and $Q$ being on the same side of $a$, we mean that the germs of $p$ and $q$ starting at $P$ and $Q$ respectively are on the same side of $a$.

LEMma 3.7. Initial [terminal] points of oriented joinable segments of $b$ are on the same side of $a$.

Proof. In fact, otherwise there would exist a path, arbitrarily close to $a$, connecting points on different sides of $a$ which is disjoint from $a$ (cf. Lemma $3.6)$.

Lemma 3.8. Let $p$ and $q \neq-p$ be oriented segments such that $q$ begins at the terminal point of $p$. Then $p$ and $q$ are not joinable.

Proof. Suppose $p$ and $q$ are joinable. Then $p$ and $q$ are one-sided and by Lemma 3.7, the initial points of $p$ and $q$ are on the same side of $a$. Hence the initial and terminal points of $p$ are on different sides of $a$. Since $p$ and $q$ are joinable, by Lemma 3.6, there exists a disk $\Delta$ with boundary consisting of $p, q$ and arcs of $a$ connecting the initial point of $p$ with the terminal point of $p$ and the terminal point of $p$ with the terminal point of $q$. In order to imagine possible configurations of $a, p$ and $q$, it is convenient to think of a rectangle with two opposite sides $p$ and $q$ such that the remaining sides are glued to different sides of $a$ in such a way that $p$ and $q$ are one-sided and 
the terminal point of $p$ coincides with the initial point of $q$. There are two possibilities to do it (see Figure 3): either the initial point of $p$ is between the end points of $q$, or the terminal point of $q$ is between the end points of $p$ (the third possibility, that the initial point of $p$ and the terminal point of $q$ coincide, is impossible since $b$ is generic).

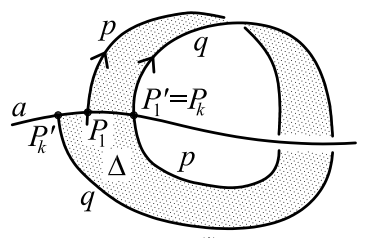

(i)

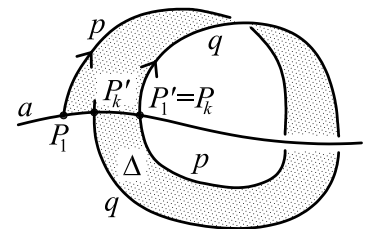

(ii)

Fig. 3. Configuration of $p, q$ and $a$-Lemma 3.8

Geometrically, it is quite clear that the situation shown in Figure 3 is not possible. In fact, this figure implies that $b$ "winds" infinitely many times along the core of a Möbius strip (it cannot turn back, because $a$ and $b$ do not cobound a bigon, and since everything is smooth there is no risk of pathologies).

In order to have a more formal argument, recall that Lemma 3.6 implies that $\bar{\Delta} \cap b$ consists of $k$ segments $P_{1} P_{1}^{\prime}, \ldots, P_{k} P_{k}^{\prime}$ of $b$ such that $P_{1} P_{1}^{\prime}=p$ and $P_{k} P_{k}^{\prime}=q$. In particular, each of the $\operatorname{arcs} P_{1} P_{k}$ and $P_{1}^{\prime} P_{k}^{\prime}$ of $a \cap \bar{\Delta}$ contains $k$ points of $b$. But this is impossible since either $P_{1} P_{k} \subset P_{1}^{\prime} P_{k}^{\prime}$ and $P_{k}^{\prime} \in P_{1}^{\prime} P_{k}^{\prime} \backslash P_{1} P_{k}$ (Figure $3(\mathrm{i})$ ), or $P_{1}^{\prime} P_{k}^{\prime} \subset P_{1} P_{k}$ and $P_{1} \in P_{1} P_{k} \backslash P_{1}^{\prime} P_{k}^{\prime}$ (Figure 3(ii)).

Definitions. By a double segment of $b$ we mean an unordered pair of two different oriented segments of $b$ which have the same initial point.

Clearly each point of $a \cap b$ determines exactly one double segment, so in particular, there are $|a \cap b|$ double segments.

Two double segments are called joinable if there exists an oriented segment $p$ in the first double segment and $q$ in the other such that $p$ and $q$ are joinable.

Lemma 3.9. Suppose $I(a, b)>1$. Then for each double segment $P$ there exists a double segment $Q \neq P$ which is not joinable to $P$.

Proof. Assume that every double segment is joinable to $P$. Let $p_{1}, p_{2}$ be oriented segments forming $P$. Since $I(a, b)>1, p_{1} \neq-p_{2}$. Let us adopt the notation of consecutive segments of $b$ as in Figure 4. We have the following

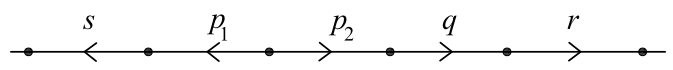

Fig. 4. Segments of $b$-Lemma 3.9

relationships: 
- $s$ and $p_{2}$ are joinable: this is because by Lemma $3.4,-p_{1}$ is not joinable to $p_{1}$ and by Lemma 3.8, it is not joinable to $p_{2}$. Therefore $s$ must be joinable either to $p_{1}$ or $p_{2}$ (since we assumed that every double segment is joinable to $P$ ). Lemma 3.8 implies that it is joinable to $p_{2}$.

- The initial and terminal points of $p_{1}$ are on the same side of $a$ : this follows by Lemma 3.7, from joinability of $s$ and $p_{2}$.

- $q$ and $p_{1}$ are joinable: this is because $-p_{2}$ is joinable neither to $p_{2}$ (Lemma 3.4) nor to $p_{1}$ (Lemma 3.8), and $q$ is not joinable to $p_{2}$ (Lemma $3.8)$.

- The initial and terminal points of $p_{2}$ are on the same side of $a$ : this follows by Lemma 3.7, from joinability of $q$ and $p_{1}$.

- $r$ and $p_{2}$ are joinable: this is because $-q$ is joinable neither to $p_{2}$ (Lemma 3.7) nor to $p_{1}$ (because $q$ is joinable to $p_{1}$ ), and $r$ is not joinable to $p_{1}$ (Lemma 3.7 ).

Figures 5(i)-(iii) show reconstruction of $a$ and $b$ due to the above properties (here, as in the proof of Lemma 3.8, one should think of joinability as a rectangle with two edges glued to $a$ ). Let $K$ be an annulus with sides:

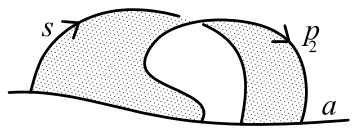

(i)

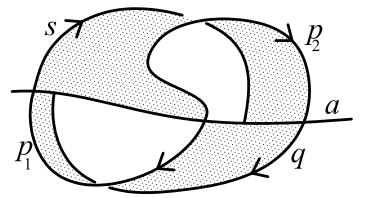

(ii)

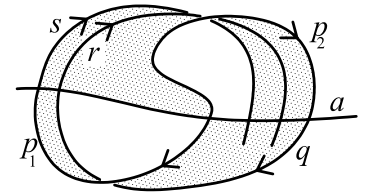

(iii)

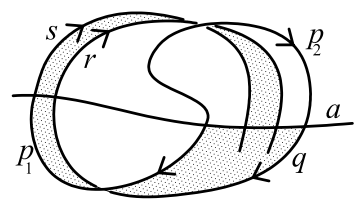

(iv)

Fig. 5. Segments of $b$-Lemma 3.9

$p_{1}, q, r, s$, the arc of $a$ connecting the initial point of $p_{2}$ with the terminal point of $s$ and the arc of $a$ connecting the terminal points of $r$ and $p_{2}$, i.e. $K$ is the shaded region in Figure 5(iv). Clearly this figure implies that $b$ winds infinitely many times along the core of $K$.

More formally, as in Lemma 3.8, Lemma 3.6 implies that each of the two sides of $K$ contained in $a$ contains the same number of points of $a \cap b$, which is impossible.

3.3. Properties of $\Gamma(a, b)$. Recall that a cycle in a graph with the set of vertices $V$ is any sequence of different edges $\left(u_{1}, u_{2}\right),\left(u_{2}, u_{3}\right), \ldots,\left(u_{k}, u_{1}\right)$, where $u_{1}, \ldots, u_{k} \in V$. 
Proposition 3.10. Every vertex in $\Gamma(a, b)$ has degree at most 2. Moreover $\Gamma(a, b)$ is a forest, i.e. it does not contain cycles (in particular there are neither loops nor multiple edges).

Proof. The first statement follows from Remark 3.2.

Suppose that there is a cycle in $\Gamma(a, b)$. By Lemma 3.4, this means that there exists a sequence $p_{1}, \ldots, p_{k}$ of oriented segments of $b$ such that $p_{1}=p_{k}$ and $p_{i}$ is adjacent to $p_{i+1}$ through a disk $\Delta_{i}$ for $i=1, \ldots, k-1$. Moreover, since every vertex has degree at most 2 , our cycle is simple (i.e. all its vertices are different), hence $\left|p_{i}\right| \neq\left|p_{j}\right|$ for $i \neq j, i, j \in\{1, \ldots, k-1\}$.

Suppose first that $k=1$, i.e. there exists a loop in $\Gamma(a, b)$ and $\Delta_{1}$ is a disk given by adjacency of $p_{1}$ to itself. Now think of $\bar{\Delta}$ as obtained by the following construction: identify two opposite sides (corresponding to $p_{1}$ ) of a rectangle (corresponding to $\Delta$ ) - this gives us an annulus, and then we have to glue the remaining sides to $a$. There are two possibilities to do it and we obtain either a torus or a Klein bottle. The first case is not possible since $p_{1}$ is one-sided and in the second case $\Gamma(a, b)=\emptyset$ (because there is only one isotopy class of generic two-sided circles on a Klein bottle-cf. Corollary A.4).

If $k>1$, since $\left|p_{i}\right| \neq\left|p_{j}\right|$ for $i \neq j, i, j \in\{1, \ldots, k-1\}$, and $\Delta_{1} \neq \Delta_{k-1}$, we have $\Delta_{i} \cap \Delta_{k-1}=\emptyset$ for $i=1, \ldots, k-2$ (cf. Remark 3.1). Therefore if $\Delta$ is an open disk obtained by applying Lemma 3.6 to the segments $p_{1}$ and $p_{k-1}$, then $\Delta \cap \Delta_{k-1}=\emptyset$. Hence we can complete the reasoning as in the case $k=1$, but with $\Delta^{\prime}=\Delta \cup p_{k-1} \cup \Delta_{k-1}$.

The following proposition shows that $\Gamma(a, b)$ could be defined not only for circles $a, b$ but for their isotopy classes. Since we will not use this result we skip its proof.

Proposition 3.11. Let $a, a^{\prime}, b, b^{\prime}$ be two-sided circles on $N$ such that $a \simeq a^{\prime}, b \simeq b^{\prime}$ and $|a \cap b|=\left|a^{\prime} \cap b^{\prime}\right|=I(a, b)$. Then $\Gamma(a, b)$ is isomorphic to $\Gamma\left(a^{\prime}, b^{\prime}\right)$.

3.4. Proof of Theorem 3.3. The theorem is trivial if $I(a, b)=0$, so assume that $I(a, b) \geq 1$.

Construction of $t_{a}^{n}(b)$. Let $S_{a}$ and $S_{b}$ be oriented regular neighbourhoods of $a$ and $b$ respectively such that $S_{a} \cup S_{b}$ is a regular neighbourhood of $a \cup b$. Define also $S_{b}^{\circ} \subset S_{b}$ to be a collar neighbourhood of $b$ and let $b^{\prime}$ be the boundary component of $S_{b}^{\circ}$ different from $b$. In particular, $b$ and $b^{\prime}$ are disjoint, isotopic and $\left|a \cap b^{\prime}\right|=|a \cap b|$. The set $S_{b}^{\circ} \cap S_{a}$ consists of $m=I(a, b)$ disjoint 4 -gons. We can label their vertices by $E_{i}, E_{i}^{\prime}, F_{i}^{\prime}, F_{i}$ for $1 \leq i \leq m$ in such a way that the following conditions are satisfied: 
(1) $E_{i} F_{i}$ and $E_{i}^{\prime} F_{i}^{\prime}$ are arcs of $b$ and $b^{\prime}$, respectively;

(2) the orientation of the 4-gon $E_{i} E_{i}^{\prime} F_{i}^{\prime} F_{i}$ induced by a cyclic ordering of vertices agrees with the orientation of $S_{a}$ (see Figure 6).

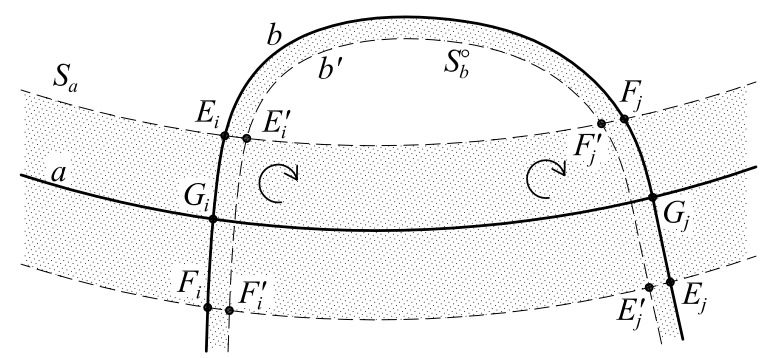

Fig. 6. Intersections of $S_{a}$ and $S_{b}^{\circ}$

Let also $G_{i}=E_{i} F_{i} \cap a$ and let us adopt the convention that unless otherwise stated the arc $E_{i} F_{i}$ (or $E_{i}^{\prime} F_{i}^{\prime}$ ) means that of the two arcs of $b$ (or $b^{\prime}$ ) with end points $E_{i}, F_{i}$ (or $E_{i}^{\prime}, F_{i}^{\prime}$ ) which is contained in $S_{a}$.

Outside $S_{a}$ the twist $t_{a}$ acts as the identity, so the circle $c=t_{a}^{n}\left(b^{\prime}\right)$ has the following properties:

(1) outside $S_{a}, c$ is equal to $b^{\prime}$;

(2) each arc of $c \cap S_{a}$ circles $|n|$ times around $S_{a}$.

Due to the above properties, each of the $m$ arcs $E_{i}^{\prime} F_{i}^{\prime}$ of $c$ crosses $b$ in $|n| m$ points (see Figure 7). In particular

$$
|c \cap b|=|n| I(a, b)^{2} .
$$

Observe that the notation is chosen in such a way that every time $c$ enters the neighbourhood $S_{a}$ through a point $E_{i}^{\prime}$, it crosses $E_{i} F_{i}$ (cf. Figure 7 ).

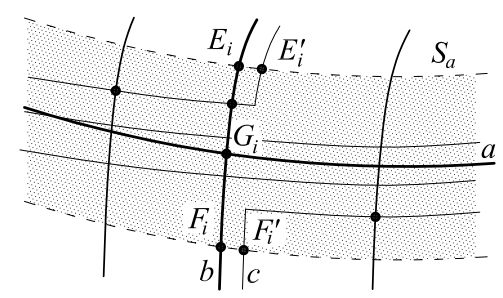

Fig. 7. Points of intersection of $c$ and $b$

Admissible circles. Now we are going to define a class of circles which contains $c$ and is closed under certain deformations (defined later).

Suppose $\gamma$ is a circle contained in $S_{a} \cup S_{b}$ and such that outside $S_{a}$, $\gamma$ consists of $m$ disjoint arcs each of which is disjoint from $b$ and has end points on different components of $\bar{P} \cap S_{a}$, where $P$ is the component of $S_{b} \backslash S_{a}$ 
containing this arc. Moreover, if we identify $S_{a}$ with $a \times[0,1]$ so that each of the arcs $b \cap S_{a}$ has constant first coordinate, then we assume that each arc of $\gamma \cap S_{a}$ is monotone with respect to the first coordinate. We then call $\gamma$ admissible. Observe that in particular, $b^{\prime}$ and $c$ are admissible.

We can extend the notion of [oriented] segments to any admissible circle $\gamma$, defining them to be components of $\overline{\gamma \backslash S_{a}}$. Moreover, since $S_{a}$ is orientable, we can speak about one-sided [two-sided] segments. In addition every oriented segment of $\gamma$ uniquely determines an oriented segment of $b$, so we have a well defined map from the set of oriented segments of $\gamma$ into the set of oriented segments of $b$. Denote this map by $\gamma_{b}$. Clearly $\gamma_{b}$ induces a map between the sets of unoriented segments of $\gamma$ and of $b$. By abuse of notation we also use the symbol $\gamma_{b}$ for this map. We will use the notion of an [oriented] segment of $b$ starting at $E_{i}$ (or $F_{i}$ ), meaning the [oriented] segment of $b$ with initial point $G_{i}$ which passes through $E_{i}\left(\right.$ or $F_{i}$ ).

Reductions of types I and II. The constructed circle $c$, in contrast to the oriented case, usually does not satisfy $I(c, b)=|c \cap b|$. However we will define two types of reduction which will enable us to deform, in a very controlled way, $c$ into a circle $d$ satisfying $I(d, b)=|d \cap b|$.

Let $p$ be an oriented one-sided segment of $b$ with initial point $G_{i}$ and terminal point $G_{j}$. Let $q$ be an oriented segment of an admissible circle $\gamma$ such that $\gamma_{b}(q)=p$. Suppose further that if we orient the arc $\widetilde{q}$ of $\gamma$ complementary to $q$ in such a way that it has the same initial and terminal points as $q$ then the first intersection point of $\gamma \cap b$ lying on $\widetilde{q}$ is on $E_{i} F_{i}$ and the last one is on $E_{j} F_{j}$. Moreover, assume that between $p$ and $q$ there are no other segments of $\gamma$ (see Figure 8). Now we see that we can push

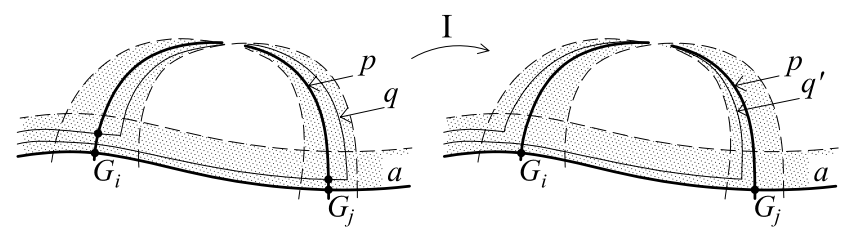

Fig. 8. Reduction of type I

the segment $q$ of $\gamma$ towards $p$ to obtain a circle $\gamma^{\prime}$ isotopic to $\gamma$ such that $I\left(\gamma^{\prime}, b\right)=I(\gamma, b)-2$. Observe also that $\gamma^{\prime}$ is admissible and $\gamma_{b}=\gamma_{b}^{\prime}$ (modulo the identification of $q$ and its deformation $q^{\prime}$ ). We call every such deformation of $\gamma$ a reduction of type $I$.

Suppose now that we have two adjacent oriented segments $p, p^{\prime}$ of $b$ with initial points $G_{i}, G_{j}$ and terminal points $G_{k}, G_{l}$ respectively. Let $q$ be an oriented segment of an admissible circle $\gamma$ such that $\gamma_{b}(q)=p^{\prime}$. Suppose further that if $\widetilde{q}$ is constructed as above then the first intersection point of 
$\gamma \cap b$ lying on $\widetilde{q}$ is on $E_{i} F_{i}$ and the last one is on $E_{k} F_{k}$. Moreover, assume that between $p$ and $q$ there are no other segments of $\gamma$ (see Figure 9). As

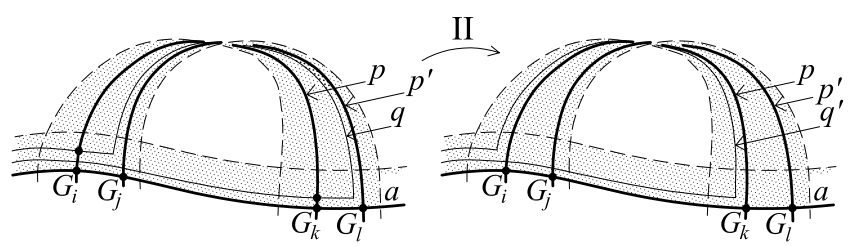

Fig. 9. Reduction of type II

before we can push $q$ towards $p$ obtaining a circle $\gamma^{\prime}$ isotopic to $\gamma$ such that $I\left(\gamma^{\prime}, b\right)=I(\gamma, b)-2$. Observe also that $\gamma^{\prime}$ is admissible and if we denote by $q^{\prime}$ the segment resulting from the deformation of $q$, we have $\gamma_{b}^{\prime}\left(q^{\prime}\right)=p$ whereas $\gamma_{b}(q)=p^{\prime}$. Outside the segments $q$ for $\gamma_{b}$ and $q^{\prime}$ for $\gamma_{b}^{\prime}$ these two maps are identical. We call every such deformation of $\gamma$ a reduction of type II.

Reducing c. Let $p=G_{i} G_{j}$ be an oriented segment of $b$. Then by the construction of $c$, there exists a unique oriented segment $q$ of $c$ with $c_{b}(q)=p$. Suppose further that $p$ and $q$ determine a reduction of type I (see Figure 8). We claim that if $q^{\prime}$ is obtained from $q$ by performing this reduction, then $p$ and $q^{\prime}$ do not allow a reduction of type I. In fact, if we orient the arc $\widetilde{q}^{\prime}$ complementary to $q^{\prime}$ in such a way that it has the same initial and terminal points as $q^{\prime}$, then the first point of $\widetilde{q}^{\prime} \cap b$ on $\widetilde{q}^{\prime}$ cannot be on $E_{i} F_{i}$ (because before $\widetilde{q}^{\prime}$ goes back to $E_{i} F_{i}$ it must intersect each $E_{l} F_{l}$ for $\left.l \neq i\right)$. Therefore if $p_{1}, \ldots, p_{k}$ are all segments of $b$ which determine a reduction of type I (with respect to $c$ ), and $c^{\prime}$ is the circle obtained from $c$ by performing these $k$ reductions, then $c^{\prime}$ admits no further reductions of type I.
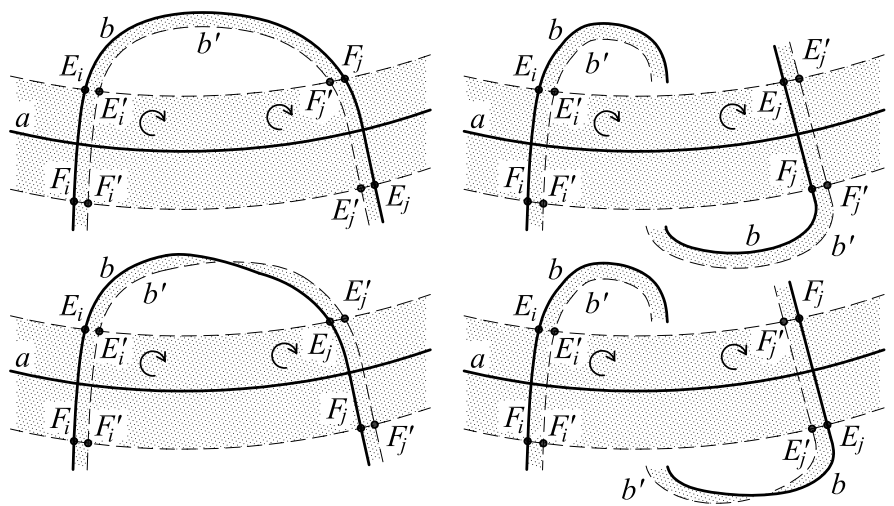

Fig. 10. Possible configurations of segments of $b$

In order to determine the number $k$, observe that if $\mathcal{E}=\left\{E_{1}, \ldots, E_{m}\right\}$ and $\mathcal{F}=\left\{F_{1}, \ldots, F_{m}\right\}$ then a segment $p$ of $b$ is one-sided if and only if both 
its end points are in $\mathcal{E}$ or $\mathcal{F}$ (see Figure 10). Moreover, if $p^{\prime}$ is a segment of $c$ with $c_{b}\left(p^{\prime}\right)=p$ then $p$ and $p^{\prime}$ determine a reduction of type I if and only if both end points of $p$ are in $\mathcal{E}$ (cf. Figure 7). Observe also that the above characterisation of one-sided and two-sided segments of $b$ in terms of their end points shows that the number of one-sided segments with end points in $\mathcal{E}$ is equal to the number of one-sided segments with end points in $\mathcal{F}$ (they alternate along $b$ ). Since the total number of one-sided segments of $b$ is $\sum_{i=1}^{u} k_{i}$, where $k_{1}, \ldots, k_{u}$ are the numbers of vertices in the connected components of $\Gamma(a, b)$, we see that $k=\frac{1}{2} \sum_{i=1}^{u} k_{i}$. Therefore

$$
\left|c^{\prime} \cap b\right|=|c \cap b|-\sum_{i=1}^{u} k_{i} .
$$

Notice also that $c^{\prime}$ is admissible and $c_{b}^{\prime}=c_{b}$ (up to the obvious identification of domains).

By Proposition 3.10, every connected component $K_{i}$ of $\Gamma(a, b)$ is a path, so every such component determines a sequence $p_{1}, \ldots, p_{k_{i}}$ ( $k_{i}$ being the number of vertices in $K_{i}$ ) of segments of $b$ such that $p_{i}$ is adjacent to $p_{i+1}$ for $i=1, \ldots, k_{i}-1$. Therefore we see that $K_{i}$ determines $1+2+\cdots+\left(k_{i}-1\right)$ reductions of $c^{\prime}$ of type II (see Figure 11). Let $d$ be the circle obtained by

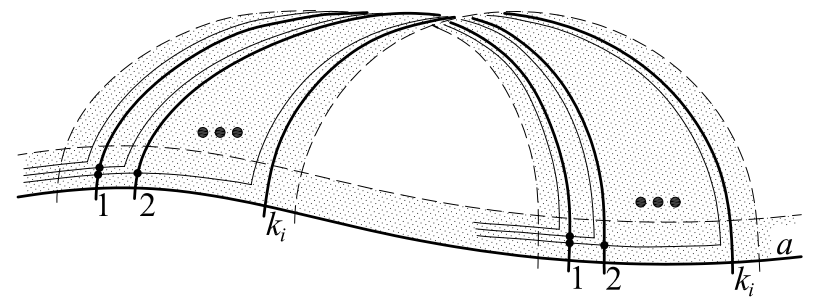

Fig. 11. Segments of $b$ and $c^{\prime}$ corresponding to $K_{i}$

performing all these reductions, in particular

$$
|d \cap b|=\left|c^{\prime} \cap b\right|-\sum_{i=1}^{u} k_{i}\left(k_{i}-1\right)=|c \cap b|-\sum_{i=1}^{u} k_{i}^{2}=|n| I(a, b)^{2}-\sum_{i=1}^{u} k_{i}^{2} .
$$

We claim that $d$ admits no further reductions. First observe that every reduction of type II is determined by two adjacent segments $p$ and $p^{\prime}$ of $b$. By the construction of $d$ at least one of the preimages $d_{b}^{-1}(p)$ or $d_{b}^{-1}\left(p^{\prime}\right)$ is empty. Hence $d$ admits no reduction of type II. In order to show that $d$ admits no reduction of type $\mathrm{I}$, suppose that $p=G_{i} G_{j}$ is an oriented one-sided segment of $b$ and $q$ is an oriented segment of $d$ such that $d_{b}(q)=p$. Denote also by $\widetilde{q}$ the arc complementary to $q$ oriented in such a way that $G_{i}$ is its initial point. By the construction of $d$ it is clear that the first point of $d \cap b$ lying on $\widetilde{q}$ cannot be on $E_{i} F_{i}$ (because before $\widetilde{q}$ goes back to $E_{i} F_{i}$ it must intersect each $E_{l} F_{l}$ for $l \neq i$ ). 
To finish the proof it is enough to show that $|d \cap b|=I(d, b)$, i.e. that $b$ and $d$ do not cobound a bigon (cf. Proposition 2.1).

Denote by $E_{1}^{d}, \ldots, E_{m}^{d}, F_{1}^{d}, \ldots, F_{m}^{d}$ the points of intersection of $d$ with the boundary of $S_{a}$ corresponding to the points $E_{1}^{\prime}, \ldots, E_{m}^{\prime}, F_{1}^{\prime}, \ldots, F_{m}^{\prime}$ of $c$ (i.e. the segment $E_{i}^{d} F_{i}^{d}$ of $d$ is the deformation of $E_{i}^{\prime} F_{i}^{\prime}$ ).

Before we proceed further we need the following corollary of Lemma 3.9.

Lemma 3.12. If $I(a, b)>1$, then for every $1 \leq i \leq m$, each of the arcs $E_{i} F_{i}$ and $E_{i}^{d} F_{i}^{d}$ intersects the set $(b \cap d) \backslash\left(E_{i} F_{i} \cap E_{i}^{d} \bar{F}_{i}^{d}\right)$.

Proof. For a fixed $i$, by Lemma 3.9, there exists a double segment $P$ which is not joinable to the double segment determined by $E_{i} F_{i}$. Assume that $P$ is determined by an arc $E_{j} F_{j}$ for some $j \neq i$ (see Figure 12). Now

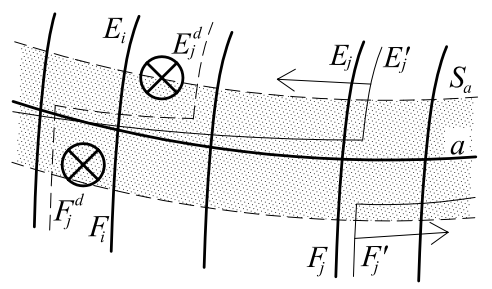

Fig. 12. Configuration of segments-Lemma 3.12

one should think that $d$ is obtained from $c$ by unwinding along adjacent segments. Since no oriented segment of $P$ is joinable to an oriented segment of the double segment $E_{i} F_{i}, E_{j}^{\prime} F_{j}^{\prime}$ cannot unwind along $E_{i} F_{i}$ and vice versa (crossed disks in Figure 12 represent obstacles to the unwinding). Hence the $\operatorname{arc} E_{j}^{d} F_{j}^{d}$ intersects $E_{i} F_{i}$ and $E_{i}^{d} F_{i}^{d}$ intersects $E_{j} F_{j}$.

Minimality of $d \cap b$. Suppose that $b$ and $d$ cobound a bigon $\Delta$ with vertices $X, Y$. Assume that $X$ is on the $\operatorname{arcs} E_{i} F_{i}, E_{j}^{d} F_{j}^{d}$, and $Y$ on $E_{k} F_{k}$, $E_{l}^{d} F_{l}^{d}$.

First consider the case $m=1$. Since there are at least two points of intersection $b \cap d$, we have $|n| \geq 2$. Observe that since there are no one-sided segments of $b$, we have $d=c$. Now there are two possibilities: either the arc $b \cap \partial \Delta$ is contained in $S_{a}$ or it passes through $E_{1}$ and $F_{1}$. Similarly, there are two possibilities for the position of the second arc of $\partial \Delta$ (see Figure 13; observe that cases (ii) and (iii) are possible only if $|n|=2$ ). In each of these cases, the path indicated in Figure 13 (running along $b$ ) connects points on different sides of $\partial \Delta$ and is disjoint from $\partial \Delta$, a contradiction.

Therefore we further assume that $I(a, b)>1$. Now the proof splits into two cases.

CASE 1: $i=k$. There are two $\operatorname{arcs}$ of $b$ joining $X$ and $Y$ : the one contained in $S_{a}$ and another one, running through $E_{i}$ and $F_{i}$. Observe that 

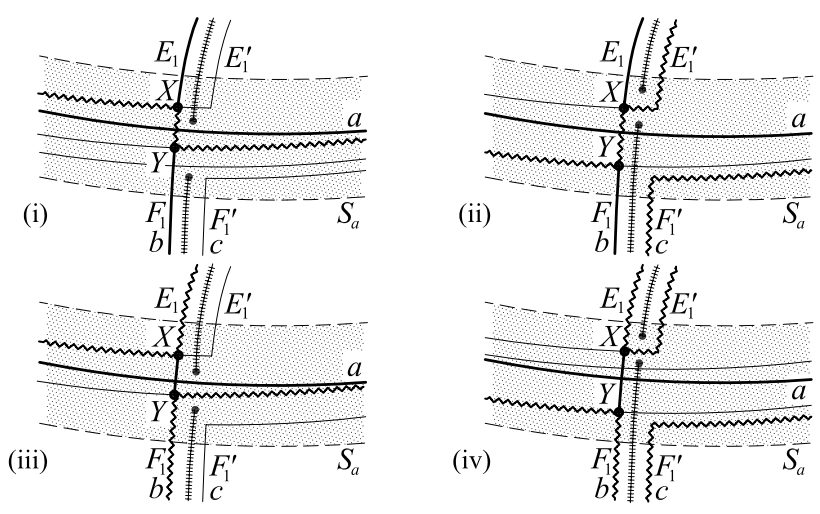

Fig. 13. Points of intersection of $c$ and $b$ if $I(a, b)=1$

only the first one can be a boundary arc of the bigon $\Delta$. This follows from the observation that by the assumption $I(a, b)>1$ and by Lemma 3.12, $X$ and $Y$ cannot be consecutive on the second of these arcs. Now depending on the position of the second boundary arc of $\Delta$, we deduce that either the boundary of $\Delta$ is a nonseparating circle, or $a$ and $b$ cobound a bigon-see Figure 14 .
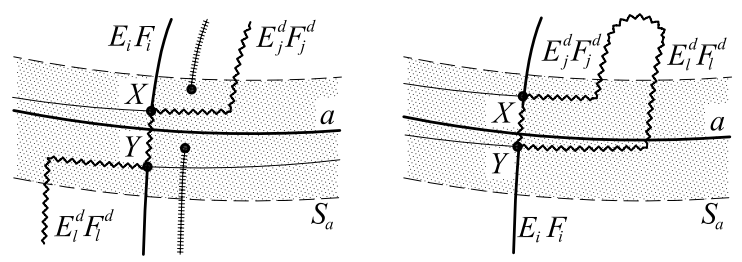

Fig. 14. The case $i=k$

CASE 2: $i \neq k$. Since $X$ and $Y$ are consecutive on $b$, there exists an arc of $b$ with end points $X$ and $Y$ whose interior is disjoint from $d$. By Lemma 3.12 , this arc outside $S_{a}$ is equal to the segment $p$ of $b$ with end points $G_{i}$ and $G_{k}$.

If $j=l$ then $a$ and $b$ would cobound a bigon (see Figure 15), so $j \neq l$.

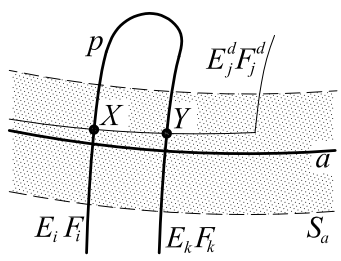

Fig. 15. The case $i \neq k, j=l$

Since $X$ and $Y$ are consecutive on $d$, there exists an arc of $d$ with end points $X$ and $Y$ whose interior is disjoint from $b$. As before, by Lemma 3.12, 
this arc outside $S_{a}$ is equal to the segment $q$ of $d$ with one end point $E_{j}^{d}$ or $F_{j}^{d}$ and the other one $E_{l}^{d}$ or $F_{l}^{d}$-see Figure 16.
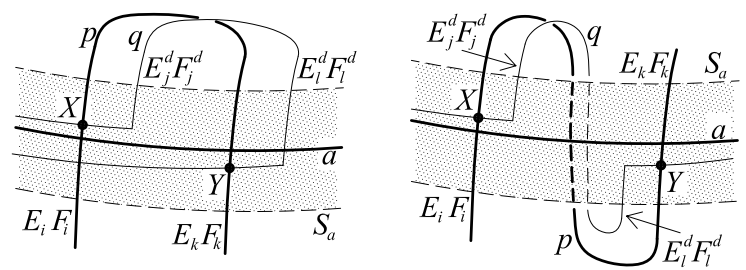

Fig. 16. The case $i \neq k, j \neq l$

First observe that $q$ is one-sided. In fact, otherwise the $\operatorname{arc} X Y$ of $\partial \Delta$ corresponding to $q$ at one end would intersect $d_{b}(q)$ and at the other would not (see Figure 10). This would imply that $d_{b}(q)=p$ and $d_{b}(q) \neq p$ at the same time - a contradiction. From this it follows that $p$ is also one-sided (otherwise $\partial \Delta$ would be one-sided).

Therefore the existence of $\Delta$ implies that if $d_{b}(q) \neq p$ then $d_{b}(q)$ and $p$ are adjacent and we can perform a reduction of type II-see Figure 17(i). In case $d_{b}(q)=p$ it is possible to perform a reduction of type I-see Figure 17(ii). Hence in both cases we obtain a contradiction with the construction of $d$.

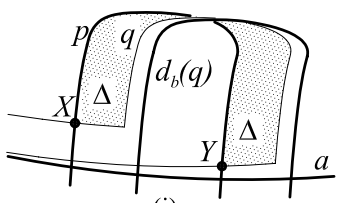

(i)

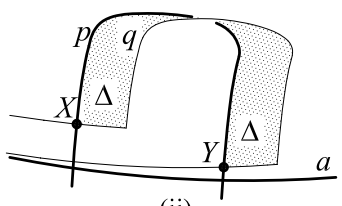

(ii)

Fig. 17. The case $i \neq k, j \neq l$

\subsection{Further remarks}

REMARK 3.13. Observe that if $\Gamma(a, b)=\emptyset$, i.e. if a regular neighbourhood of $a \cup b$ is orientable, then $c=d$ and the proof of Theorem 3.3 works without the assumption that $a$ and $b$ are generic (Lemma 3.12 is not needed). In particular, if $I(a, b)>0$, Theorem 3.3 implies that $t_{a} \neq 1$, hence $a$ is generic.

Proposition 3.14. Let $n \neq 0$ be an integer. Then

(1) $I\left(t_{a}^{n}(b), b\right)=|n|$ if $I(a, b)=1$;

(2) $I\left(t_{a}^{n}(b), b\right) \geq I(a, b)$;

(3) $I\left(t_{a}^{n}(b), b\right) \geq(|n|-1) I(a, b)^{2}+2 I(a, b)-2$.

In particular, if $I(a, b) \neq 0$, then $I\left(t_{a}^{n}(b), b\right)>0$. 
Proof. The assertion is trivial for $I(a, b)=0$, so let $I(a, b) \geq 1$. By the proof of Theorem 3.3, $I\left(t_{a}^{n}(b), b\right)=|d \cap b|$. If $I(a, b)=1$ then $|d \cap b|=|c \cap b|$ $=|n|$, which proves (1). The inequality (2) follows from (1) if $I(a, b)=1$, and if $I(a, b) \geq 2$ then by Lemma 3.12, $|d \cap b| \geq I(a, b)$.

In order to prove (3), first observe that if $k_{1}, \ldots, k_{u}$ are as in the statement of Theorem 3.3, then by (2), $\sum_{i=1}^{u} k_{i}^{2}<I(a, b)^{2}$ (otherwise $I\left(t_{a}(b), b\right)=0$ ). Therefore $\Gamma(a, b)$ is not a path with $I(a, b)$ vertices, i.e. $u>1$. Now it is an easy exercise that if $a$ and $b$ are positive integers such that $a+b=m$, then $a^{2}+b^{2} \leq 1+(m-1)^{2}$. Hence

$$
\sum_{i=1}^{u} k_{i}^{2} \leq k_{1}^{2}+\left(\sum_{i=2}^{u} k_{i}\right)^{2} \leq 1+(I(a, b)-1)^{2} .
$$

By Theorem 3.3, the above inequality yields (3).

\section{Algebraic properties of twists}

Lemma 4.1. Assume that $s+r \geq 2$ if $g=2$, and let $a_{1}, \ldots, a_{u}$ be generic two-sided circles on $N=N_{g, r}^{s}$ such that:

(1) $a_{i} \cap a_{j}=\emptyset$ if $i \neq j$;

(2) $a_{i}$ is isotopic neither to $a_{j}$ nor to $a_{j}^{-1}$ if $i \neq j$;

(3) none of the $a_{i}$ is isotopic to a boundary component of $N$;

(4) if we cut $N$ along those $a_{i}$ which separate $N$, then every component homeomorphic to a Klein bottle with one boundary component is disjoint from $a_{1}$.

Then there exists a generic two-sided circle $b$ such that $a_{i} \cap b=\emptyset$ if $i \neq 1$, and $\left|a_{1} \cap b\right|=I\left(a_{1}, b\right)>0$.

Proof. Let $N^{\prime}$ be the connected component of $N \backslash \bigcup_{j=2}^{u} a_{j}$ containing $a_{1}$. Clearly it is enough to construct a generic two-sided circle $b$ on $N^{\prime}$ such that $\left|a_{1} \cap b\right|=I\left(a_{1}, b\right)>0$. Now if we cut $N^{\prime}$ open along $a_{1}$ we obtain a surface $N^{\prime \prime}$ with two more boundary components; denote them by $\alpha_{1}$ and $\alpha_{2}$. Moreover, if we fix the orientation of $a_{1}$, then $\alpha_{1}$ and $\alpha_{2}$ inherit orientations from $a_{1}$. Consider two cases:

CASE 1: $N^{\prime \prime}$ is connected. If $N^{\prime \prime}$ is nonorientable then we can represent $N^{\prime \prime}$ as a connected sum of an oriented surface and a number of projective planes. Now depending on mutual orientations of $\alpha_{1}$ and $\alpha_{2}$, one of the two curves indicated in Figure 18 projects to a two-sided circle $b$ on $N^{\prime}$ (the shaded disk in Figure 18 represents a crosscap on $N^{\prime \prime}$ ).

If $N^{\prime \prime}$ and $N^{\prime}$ are orientable then the construction of $b$ is shown in Figure 19(i). If $N^{\prime \prime}$ is orientable and $N^{\prime}$ is nonorientable then either $N^{\prime \prime}$ has genus at least 1 or by assumption, it has at least two punctures/boundary components 

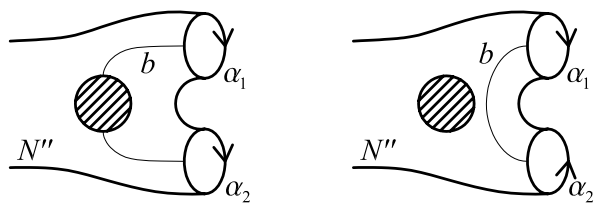

Fig. 18. Construction of $b$ if $N^{\prime \prime}$ is nonorientable

different from $\alpha_{1}$ and $\alpha_{2}$. The construction of $b$ in each of these cases is shown in Figures 19(ii) and 19(iii) respectively.

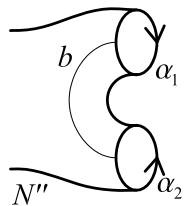

(i)

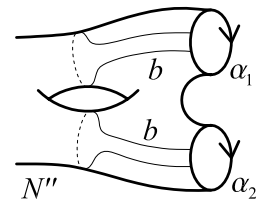

(ii)

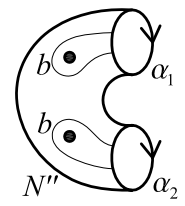

(iii)

Fig. 19. Construction of $b$ if $N^{\prime \prime}$ is orientable

CASE 2: $N^{\prime \prime}$ is disconnected. Let $M_{1}$ and $M_{2}$ be connected components of $N^{\prime \prime}$ such that $\alpha_{k}$ is a boundary component of $M_{k}, k=1,2$. Observe that for $k=1,2$, we have:

(1) if $M_{k}$ has genus 0 then it has at least two punctures/boundary components different from $\alpha_{k}$ (since $a_{1}$ is generic and isotopic neither to a boundary component of $N$ nor to any of $a_{j}^{ \pm 1}, j \geq 2$ );

(2) if $M_{k}$ is nonorientable of genus 1 , then it has at least one puncture or a boundary component different from $\alpha_{k}$ (since $a_{1}$ is generic);

(3) if $M_{k}$ is orientable of genus at least 1 or nonorientable of genus at least 2 , then $M_{k}$ is a connected sum of a torus/Klein bottle with boundary component $\alpha_{k}$ and some other surface.

Therefore, in any case we can construct an arc $\beta_{k}$ on each of $M_{k}, k=1,2$, such that projections of $\beta_{1}$ and $\beta_{2}$ onto $N^{\prime}$ give a two-sided circle $b$ such that $\left|a_{1} \cap b\right|=I\left(a_{1}, b\right)=2$ (see Figure 20).
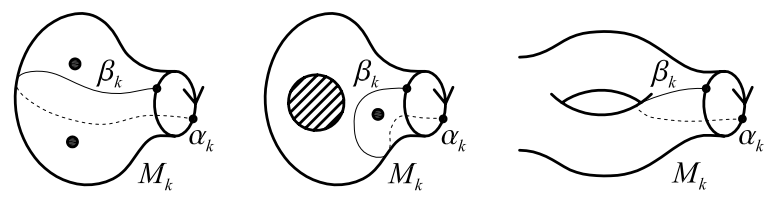

Fig. 20. Construction of $b$ if $N^{\prime \prime}$ is disconnected

Observe that in each case, $\Gamma\left(a_{1}, b\right)=\emptyset$, hence by Remark $3.13, b$ is generic. 
REMARK 4.2. It is easy to prove that if $a$ is a generic two-sided circle on a Klein bottle with one boundary component, which is not isotopic to the boundary, then $a$ is nonseparating (cf. Lemma A.1 and its proof). Therefore, if $a_{1}, \ldots, a_{u}$ are generic two-sided circles on a closed surface, satisfying all assumptions of the above lemma but (4), then $a_{1}$ is nonseparating and $a_{j}$ is separating for some $j>1$.

For each nonorientable surface $N$, let $\widehat{N}$ be the surface obtained by gluing a torus minus a disk to each boundary component of $N$. Then $\widehat{N}$ has no boundary and the following, very useful, property (cf. Proposition 3.5 of [10]):

Proposition 4.3. Suppose $a$ and $b$ are circles on $N$. Then $a$ is isotopic to $b$ in $N$ if and only if they are isotopic in $\widehat{N}$.

It is an easy observation that the only nontrivial Dehn twist on a Klein bottle has order 2. The next proposition shows that except for this example, Dehn twists about disjoint circles generate a free abelian group (we will use this result in the proof of Theorem 6.2).

Proposition 4.4. Suppose $r+s>0$ if $g=2$, and let $a_{1}, \ldots, a_{u}$ be generic two-sided circles on $N=N_{g, r}^{s}$ such that $a_{i} \cap a_{j}=\emptyset$ if $i \neq j$, and $a_{i}$ is isotopic neither to $a_{j}$ nor to $a_{j}^{-1}$ if $i \neq j$. Consider the function $h: \mathbb{Z}^{u} \rightarrow \mathcal{M}(N)$ defined by

$$
h\left(n_{1}, \ldots, n_{u}\right)=t_{a_{1}}^{n_{1}} \cdots t_{a_{u}}^{n_{u}} .
$$

Then $h$ is an injective homomorphism.

Proof. Clearly $h$ is a homomorphism, so let us prove that it is injective. For $N$ being a Klein bottle with a puncture the assertion follows from Proposition A.3 and Theorem A.5, so assume that $N$ is not a Klein bottle with a puncture. Suppose $t_{a_{1}}^{n_{1}} \cdots t_{a_{u}}^{n_{u}}=1$ in $\mathcal{M}(N)$. Clearly $t_{a_{1}}^{n_{1}} \cdots t_{a_{u}}^{n_{u}}=1$ also in $\mathcal{M}(\widehat{N})$, where $\widehat{N}$ is the surface described above. Without loss of generality we can assume that the first $k$ of the circles $a_{1}, \ldots, a_{u}$ are separating on $\widehat{N}$ and the remaining ones are not. We will prove by induction on $i$ that $n_{i}=0$. Suppose that $n_{j}=0$ for $j<i$. By Proposition 4.3 and by Remark 4.2 , the circles $a_{i}, a_{i+1}, \ldots, a_{u}$ and the surface $\widehat{N}$ satisfy the assumptions of Lemma 4.1. Therefore, there exists a circle $b$ on $\widehat{N}$ such that $a_{j} \cap b=\emptyset$ for $j>i$ and $\left|a_{i} \cap b\right|=I\left(a_{i}, b\right)>0$. Now if $n_{i} \neq 0$, Proposition 3.14 yields

$$
0=I(b, b)=I\left(t_{a_{i}}^{n_{i}} \cdots t_{a_{u}}^{n_{u}}(b), b\right)=I\left(t_{a_{i}}^{n_{i}}(b), b\right)>0 .
$$

Hence $n_{i}=0$, which completes the proof.

Corollary 4.5. Suppose $r+s>0$ if $g=2$, and let a be a generic two-sided circle on $N=N_{g, r}^{s}$. Then the Dehn twist $t_{a}$ has infinite order in $\mathcal{M}(N)$. 
If $a$ and $b$ are circles on an orientable surface and $j, k$ nonzero integers, then it is known (cf. Theorems 3.14-3.15 of [6]) that:

(1) $t_{a}^{j}=t_{b}^{k}$ if and only if $a \simeq b$ and $j=k$;

(2) $t_{a}^{j} t_{b}^{k}=t_{b}^{k} t_{a}^{j}$ if and only if $I(a, b)=0$.

Moreover if $a \not b^{ \pm 1}$ then

(3) $t_{a}^{j} t_{b}^{k} t_{a}^{j}=t_{b}^{k} t_{a}^{j} t_{b}^{k}$ if and only if $I(a, b)=1$ and $j=k= \pm 1$.

Clearly the "if" clauses of (1) and (2) also hold on nonorientable surfaces. In case (3) observe that if $a$ and $b$ are two-sided circles on a nonorientable surface and $|a \cap b|=I(a, b)=1$, then the regular neighbourhood of $a \cup b$ is a torus with one boundary component, so it makes sense to assume that the orientations of regular neighbourhoods $S_{a}, S_{b}$ of $a$ and $b$ agree. Under this assumption also the "if" clause of (3) holds (it is just a braid relation).

The next three propositions show that under some obvious assumptions, also the "only if" clauses of the above statements hold on nonorientable surfaces.

Proposition 4.6. Let $a$ and $b$ be generic two-sided circles on $N=N_{g, r}^{s}$. If $j$ and $k$ are nonzero integers such that $t_{a}^{j}=t_{b}^{k}$, then a is isotopic to $b^{ \pm 1}$. Moreover if $r+s>0$ for $g=2$ and the orientations of regular neighbourhoods of $a$ and $b$ are such that $t_{a}=t_{b}$, then $j=k$.

Proof. If $I(a, b) \geq 1$ then by Proposition 3.14, $I\left(t_{a}^{j}(b), b\right)>0$ and $I\left(t_{b}^{k}(b), b\right)=I(b, b)=0$. Therefore $I(a, b)=0$.

Suppose $a$ is not isotopic to $b^{ \pm 1}$. By Proposition 4.3, $a$ is not isotopic to $b^{ \pm 1}$ in $\widehat{N}$. Since on a Klein bottle or a Klein bottle with one puncture there is only one generic two-sided circle (up to isotopy and reversing orientationcf. Proposition A.3 and Corollary A.4), $\widehat{N}$ is neither of these surfaces. Now either $a_{1}=a, a_{2}=b$ or $a_{1}=b, a_{2}=a$ satisfy the assumptions of Lemma 4.1 (cf. Remark 4.2). In the first case we have a circle $c$ on $\widehat{N}$ such that by Proposition 3.14, $I\left(t_{a}^{j}(c), c\right)>0$ and $I\left(t_{b}^{k}(c), c\right)=I(c, c)=0$. Hence $t_{a}^{j} \neq t_{b}^{k}$. The second case can be handled in exactly the same way.

The last statement follows from Corollary 4.5.

Proposition 4.7. Let $a$ and $b$ be generic two-sided circles on $N$. If $j$ and $k$ are nonzero integers such that $t_{a}^{j} t_{b}^{k}=t_{b}^{k} t_{a}^{j}$, then $I(a, b)=0$.

Proof. The assertion is trivial for a Klein bottle (cf. Corollary A.4), so assume that $N$ is not a Klein bottle. If $c=t_{b}^{k}(a)$ then $t_{c}^{j}=t_{b}^{k} t_{a}^{j} t_{b}^{-k}=t_{a}^{j}$. By Corollary 4.5, $1 \neq t_{a}^{j}=t_{c}^{j}$, hence $c$ is generic. Therefore, by Proposition 4.6, $c$ is isotopic to $a^{ \pm 1}$. If we assume that $I(a, b)>0$ then by Proposition 3.14, $0=I(c, a)=I\left(t_{b}^{k}(a), a\right)>0-$ a contradiction. 
Proposition 4.8. Let $a$ and $b$ be generic two-sided circles on $N=N_{g, r}^{s}$ such that $a \neq b^{ \pm 1}$. If $j$ and $k$ are nonzero integers such that $t_{a}^{j} t_{b}^{k} t_{a}^{j}=t_{b}^{k} t_{a}^{j} t_{b}^{k}$, then $I(a, b)=1$. Moreover, if $|a \cap b|=I(a, b)$ and the orientations of regular neighbourhoods of $a$ and $b$ agree, then $j=k= \pm 1$.

Proof. Since there is only one isotopy class of circles on a Klein bottle (cf. Corollary A.4), $r+s>0$ if $g=2$. Moreover, we can assume that $|a \cap b|=I(a, b)$. If $I(a, b)=0$ then $t_{a}^{j}=t_{b}^{k}$, and by Proposition 4.6, $a \simeq b^{ \pm 1}$. Therefore $I(a, b)>0$. If $c=t_{a}^{j} t_{b}^{k}(a)$ then

$$
t_{c}^{j}=t_{t_{a}^{j} t_{b}^{k}(a)}^{j}=\left(t_{a}^{j} t_{b}^{k}\right) t_{a}^{j}\left(t_{a}^{j} t_{b}^{k}\right)^{-1}=t_{b}^{k} .
$$

Hence by Corollary 4.5, $c$ is generic, and by Proposition 4.6, $c \simeq b^{ \pm 1}$. This gives

$$
I(a, b)=I\left(t_{b}^{k}(a), b\right)=I\left(t_{a}^{j} t_{b}^{k}(a), t_{a}^{j}(b)\right)=I\left(c, t_{a}^{j}(b)\right)=I\left(b, t_{a}^{j}(b)\right) .
$$

Therefore by inequality (3) of Proposition 3.14,

$$
I(a, b) \geq(|j|-1) I(a, b)^{2}+2 I(a, b)-2 .
$$

This easily implies that $I(a, b) \in\{1,2\}$.

Suppose first that $I(a, b)=2$. If $\Gamma(a, b)=\emptyset$ then by Theorem 3.3,

$$
I\left(b, t_{a}^{j}(b)\right)=|j| I(a, b)^{2} \geq 4,
$$

contrary to (4.2). Therefore $\Gamma(a, b)$ has two vertices. This implies that the regular neighbourhood of $a \cup b$ is a Klein bottle with two boundary components, i.e. the configuration of $a, b$ and their regular neighbourhood is as in the left-hand part of Figure 21. The right-hand part of the same figure
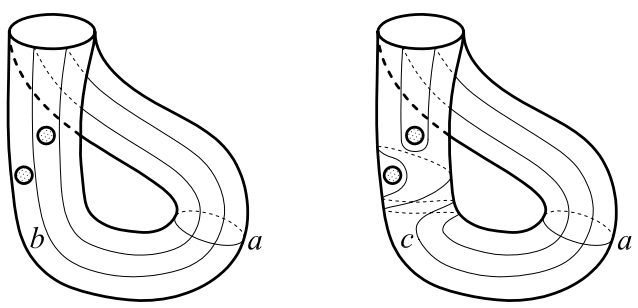

Fig. 21. Circles $a, b$ and $c=t_{a}^{j} t_{b}^{k}(a)$-Lemma 4.8

shows the circle $c=t_{a}^{j} t_{b}^{k}(a)$ (strictly speaking, since we have an ambiguity in the choice of orientations of neighbourhoods of $a$ and $b$, it is one of the possible circles $c=t_{a}^{j} t_{b}^{k}(a)$; however, other choices yield similar pictures). In particular $\Gamma(a, c)=\emptyset$ and by Theorem 3.3,

$$
I\left(c, t_{a}^{j}(c)\right)=|j| I(a, c)^{2} \geq 4,
$$

contradicting (4.2). 
Therefore $I(a, b)=1$, and by statement (1) of Proposition 3.14,

$$
I\left(b, t_{a}^{j}(b)\right)=|j| .
$$

Hence by (4.2), $|j|=1$. Now if the orientations of neighbourhoods of $a$ and $b$ agree, then $t_{c}=t_{b}$. Therefore by (4.1) and Proposition 4.6, $j=k= \pm 1$.

5. Pantalon \& skirt decompositions. To decompose nonorientable surfaces, besides standard pantalons of type I-III (see Figure 22 and Section 4 of [10]), we need two more surfaces, namely a Möbius strip $N_{1,1}^{1}$ with one puncture and a Möbius strip $N_{1,2}$ with an open disk removed, which we call (nonorientable) skirts of type I and II, respectively. The mapping class group

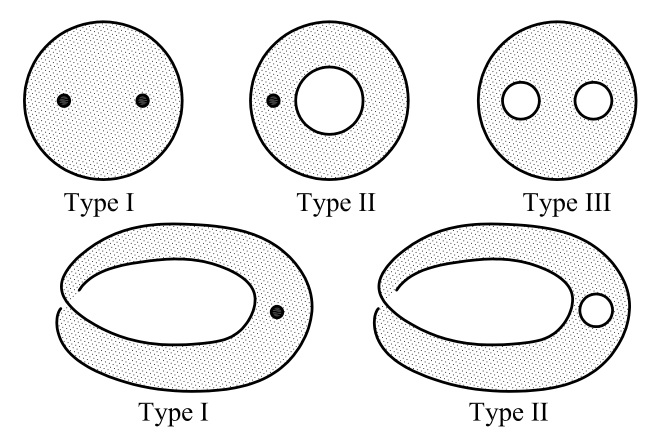

Fig. 22. Different types of pantalons and skirts

of a skirt of type II is generated by the boundary twists, and the mapping class group of a skirt of type I is generated by a puncture slide $v$ such that $v^{2}$ is a twist about the boundary component.

A decomposition of a surface into pantalons and skirts is called a $P-S$ decomposition. A P-S decomposition is called separating if each of the circles defining it is a boundary of two different pantalons/skirts.

The reason for considering separating P-S decompositions is that if we know that some diffeomorphism $f: N \rightarrow N$ preserves such a decomposition, then from the structure of the mapping class groups of pantalons/skirts we can conclude that $f$ is of a very simple form. This remark will be of great importance in the proof of Theorem 6.2.

For precise definitions of pantalons of type I-III and a pantalon decomposition, we refer the reader to [10].

The Euler characteristic of a pantalon or skirt is -1 . Therefore, none of the surfaces: $N_{1, r}^{s}$ with $r+s \leq 1, N_{1}^{2}$ nor $N_{2}$ admits a P-S decomposition. Apart from these exceptions, every nonorientable surface admits a P-S decomposition. Let us now specify some such decompositions:

- Projective plane $N_{1, r}^{s}$ with $r+s \geq 2$ and $(r, s) \neq(0,2)$. If $N$ is not a skirt, we cut off a Möbius strip with a puncture/boundary component; 
there remains a disk with at least two punctures/boundary components and we can decompose it into pantalons. The resulting decomposition is separating.

- Klein bottle $N_{2, r}^{s}$ with $r+s \geq 1$. We cut $N$ into pantalons of type II and III. If $r+s \geq 2$, this decomposition is separating.

- Nonorientable surface $N_{g, r}^{s}$ with $g \geq 3$ odd. We decompose $N$ into one skirt of type II and a number of pantalons of type II and III (see Figure 23 ; the shaded disk represents a crosscap). If $g \geq 5$ or $r+s \geq 1$, this decomposition is separating.

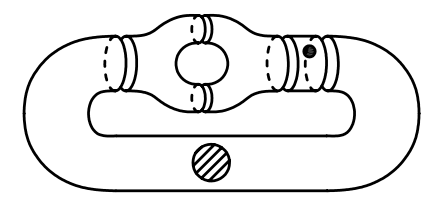

Fig. 23. P-S decomposition if the genus is odd

- Nonorientable surface $N_{g, r}^{s}$ with $g \geq 4$ even. We decompose $N$ into two pantalons of type III and a number of pantalons of type II and III (see Figure 24). This decomposition is separating.

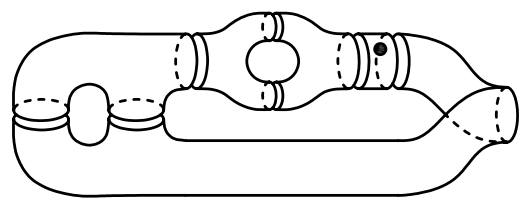

Fig. 24. P-S decomposition if the genus is even

In the following, by a P-S decomposition we will always mean one of the decompositions listed above.

6. Centralisers of subgroups generated by twists. Let $\mathcal{T}(N)$ be the twist subgroup of $\mathcal{M}(N)$, i.e. the subgroup of $\mathcal{M}(N)$ generated by all Dehn twists. In the case of a closed nonorientable surface, $\mathcal{T}(N)$ is a subgroup of index 2 (cf. [9]). If $g \geq 7$ then the index of $\mathcal{T}\left(N_{g}^{s}\right)$ is $2^{s+1} s$ ! (cf. Corollary 6.2 of $[8])$.

We now compute the centraliser $\mathcal{Z}=\mathcal{Z}_{\mathcal{M}(N)}(\mathcal{T}(N))$. This will allow us to compute the centre of $\mathcal{M}(N)$.

Observe that, as in the orientable case, boundary twists are central in $\mathcal{M}(N)$. We are going to prove that up to a finite number of exceptions, there are no other elements of $\mathcal{M}(N)$ which centralise $\mathcal{T}(N)$.

Before we state the main theorem, we need to consider some exceptional cases. 
The mapping class group of a projective plane and of a Möbius strip is trivial (cf. Theorem 3.4 of [3]).

The projective plane with one puncture, a skirt of type I, a skirt of type II and the Klein bottle have abelian mapping class groups (respectively $\left.\mathbb{Z}_{2}, \mathbb{Z}, \mathbb{Z} \times \mathbb{Z}, \mathbb{Z}_{2} \times \mathbb{Z}_{2}\right)$ so $\mathcal{Z}$ is equal to $\mathcal{M}(N)$.

If $N$ is a projective plane with two punctures, then $\mathcal{T}(N)$ is trivial, so $\mathcal{Z}$ is equal to $\mathcal{M}(N)$, i.e. to the dihedral group $D_{4}$ (of order 8) (cf. Corollary 4.6 of [8]).

If $N$ is a Klein bottle with one puncture or a Klein bottle with one boundary component then the description of $\mathcal{Z}$ follows from Corollaries A.6 and A.8.

We will now examine the case of a closed nonorientable surface $N$ of genus 3. $N$ has a double cover $\widetilde{N}$ which is an orientable surface of genus 2 . Suppose that $\widetilde{N}$ is embedded in $\mathbb{R}^{3}$ in such a way that it is invariant under reflections in the $x y, y z$ and $z x$ planes (see Figure 25 ). Let $J \in \mathcal{M}(\widetilde{N})$ be

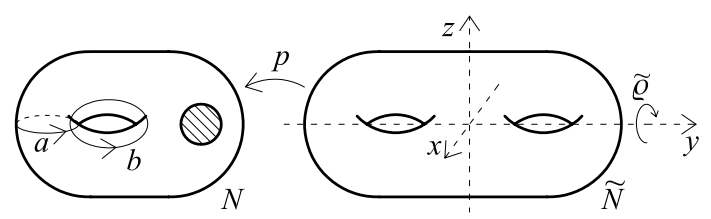

Fig. 25. Nonorientable surface of genus 3 and its double cover

the isotopy class of a diffeomorphism $j: \widetilde{N} \rightarrow \widetilde{N}$ induced by the central symmetry of $\mathbb{R}^{3}:(x, y, z) \mapsto(-x,-y,-z)$. By [1], $\mathcal{M}(N)$ is isomorphic to the quotient group $\mathcal{S}(\widetilde{N}) /\langle J\rangle$, where $\mathcal{S}(\widetilde{N})$ is the centraliser of $J$ in $\mathcal{M}(\widetilde{N})$. Moreover, this isomorphism is induced by the projection $p: \widetilde{N} \rightarrow \widetilde{N} /\langle j\rangle$, where $\widetilde{N} /\langle j\rangle$ is the orbit space, which from now on will be our model for $N$. Let $\widetilde{\varrho} \in \mathcal{M}(\widetilde{N})$ be the hyperelliptic involution, i.e. the isotopy class of a diffeomorphism induced by the half turn about the $y$-axis (see Figure 25). Since $\widetilde{\varrho}$ is central, it induces a central element $\varrho$ of $\mathcal{M}(N) \cong \mathcal{S}(\widetilde{N}) /\langle J\rangle$. Observe that if $a$ is a circle on $N$ as in Figure 25, then $\varrho(a)=a^{-1}$ and $\varrho$ preserves the local orientation of a neighbourhood of $a$.

Now let $h: N \rightarrow N$ represent an element of the centraliser $\mathcal{Z} \subseteq \mathcal{M}(N)$ of the twist subgroup. Since $t_{h(a)}=h t_{a} h^{-1}=t_{a}$, Proposition 4.6 implies that $h(a)$ is isotopic to $a^{ \pm 1}$. So we can assume that $h(a)=a^{ \pm 1}$. Moreover, $h$ must preserve the local orientation of a neighbourhood of $a$. Therefore we can choose $\varepsilon \in\{0,1\}$ such that $h \varrho^{\varepsilon}$ is isotopic to the identity in a neighbourhood of $a$. Now we can cut $N$ open along $a$, and conclude from the mapping class group of the skirt of type II that $h \varrho^{\varepsilon}=t_{a}^{k}$ for some integer $k$. Now by Lemma 4.1, there exists a two-sided generic circle $b$ such that $I(a, b)>0$ 
(see Figure 25). Since $t_{a}^{k}=h \varrho^{\varepsilon}$ commutes with the twist $t_{b}$, Proposition 4.7 implies that $k=0$. Therefore we have proved the following:

Proposition 6.1. Let $N$ be a closed nonorientable surface of genus 3 . The centre of $\mathcal{M}(N)$ is equal to the centraliser $\mathcal{Z}$ of the twist subgroup and is generated by the involution $\varrho$.

Now we are ready to prove the general result concerning the centraliser $\mathcal{Z}$.

Theorem 6.2. Suppose that $g+r+s \geq 4$ and let $c_{1}, \ldots, c_{r}$ be the boundary curves of $N=N_{g, r}^{s}$. Then the centraliser $\mathcal{Z}$ of the twist subgroup is equal to the centre of $\mathcal{M}(N)$. Moreover, $\mathcal{Z}$ is generated by $t_{c_{1}}, \ldots, t_{c_{r}}$ and is isomorphic to $\mathbb{Z}^{r}$.

Proof. Since the proof follows the lines of the proof of Theorem 5.6 of [10], we only sketch it.

The isomorphism $\left\langle t_{c_{1}}, \ldots, t_{c_{r}}\right\rangle \cong \mathbb{Z}^{r}$ follows from Proposition 4.4, so it is enough to prove that $\mathcal{Z}=\left\langle t_{c_{1}}, \ldots, t_{c_{r}}\right\rangle$.

Let $a_{1}, \ldots, a_{u}$ be the circles defining a separating P-S decomposition of $N$ (cf. Section 5). If $h \in \mathcal{Z}$ then $t_{h\left(a_{i}\right)}=h t_{a_{i}} h^{-1}=t_{a_{i}}$, hence by Proposition 4.6, $h\left(a_{i}\right) \simeq a_{i}^{ \pm 1}$ for $i=1, \ldots, u$. Now we can assume that in fact $h\left(a_{i}\right)=a_{i}^{ \pm 1}$ (cf. Proposition 3.10 of [10]), hence $h$ permutes pantalons/skirts.

First suppose that $h$ interchanges some two components $M_{1}$ and $M_{2}$ of the P-S decomposition.

If $M_{1}$ and $M_{2}$ are both pantalons of type II glued along a circle $a_{j}$, then the remaining boundary curves $a_{k} \subset M_{1}$ and $a_{l} \subset M_{2}$ must be glued together. In fact, since $h\left(a_{i}\right)=a_{i}^{ \pm 1}$ for every $i$, and $h$ interchanges $a_{k}^{ \pm 1}$ and $a_{l}^{ \pm 1}$, we have $a_{k}=a_{l}^{ \pm 1}$. Therefore $N$ is a Klein bottle with two punctures. Observe that $h$ must preserve orientations of regular neighbourhoods of $a_{j}$ and $a_{k}$ and this is possible only if $h$ does not interchange $M_{1}$ and $M_{2}$.

If $M_{1}$ and $M_{2}$ are both pantalons of type III, then as before we argue that $N$ is a closed nonorientable surface of genus 4 and $h$ does not interchange $M_{1}$ and $M_{2}$.

Observe that by our choice of P-S decompositions (cf. Section 5), and since $N$ is nonorientable, $M_{1}$ and $M_{2}$ can be neither a pantalon of type I nor a skirt.

Thus we have proved that $h$ maps every pantalon/skirt onto itself. Moreover, since $h$ centralises their boundary twists, the restriction of $h$ to each pantalon preserves its orientation.

If $N \neq N_{1}^{s}$ then the P-S decomposition of $N$ contains neither a pantalon of type I nor a skirt of type I (cf. Section 5). By the structure of the mapping class groups of pantalons of type II/III and skirts of type II,

$$
h=t_{a_{1}}^{\alpha_{1}} \cdots t_{a_{u}}^{\alpha_{u}} \gamma_{c_{1}}^{\gamma_{1}} \cdots t_{c_{r}}^{\gamma_{r}}
$$


Now for each fixed $1 \leq i \leq u$, by Lemma 4.1, there exists a generic two-sided circle $b$ such that $I\left(a_{i}, b\right)>0$ and $a_{j} \cap b=\emptyset$ for $j \neq i$. Therefore $t_{b}$ commutes with $t_{a_{j}}$ for $j \neq i$. It also commutes with all $t_{c_{i}}$ and with $h$, hence it commutes with $t_{a_{i}}^{\alpha_{i}}$. By Proposition 4.7, this yields $\alpha_{i}=0$, which completes the proof in this case.

It remains to consider the case of $N$ being a projective plane with $s \geq 3$ punctures $\Sigma=\left\{P_{1}, \ldots, P_{s}\right\}$. For each $1 \leq i \leq s$, there exists a two-sided circle $c$ on $N$ such that $N \backslash c$ has two components, one of which is a Möbius strip with a puncture $P_{i}$, and the other is a disk with $s-1$ punctures. Since $h$ centralises the twist about $c$, it satisfies $h(c) \simeq c^{ \pm 1}$. Because the components of $N \backslash c$ are not homeomorphic, $h$ cannot interchange them, so in particular, $h\left(P_{i}\right)=P_{i}$. Therefore $h$ fixes $\Sigma$ pointwise.

Now the P-S decomposition of $N$ consists of one skirt of type I and a number of pantalons of type I and II; assume that $a_{i}$ is the circle which cuts off the skirt. Since $h$ preserves the orientation of every pantalon, by the structure of the mapping class groups of the pantalons and of the skirt,

$$
h=v^{k} t_{a_{2}}^{\alpha_{2}} t_{a_{3}}^{\alpha_{3}} \cdots t_{a_{u}}^{\alpha_{u}}
$$

where $v$ is a boundary slide. Then

$$
h^{2}=t_{a_{1}}^{k} t_{a_{2}}^{2 \alpha_{2}} \cdots t_{a_{u}}^{2 \alpha_{u}} .
$$

Now a similar argument as before yields $k=\alpha_{2}=\alpha_{3}=\cdots=\alpha_{u}=0$.

Corollary 6.3. Suppose $g+s \geq 4$. Then the centre of $\mathcal{M}\left(N_{g}^{s}\right)$ is trivial.

\section{Appendix A. Mapping class group of a Klein bottle with one puncture/boundary component}

A.1. Mapping class group of a Klein bottle with one puncture. For the rest of this subsection let $N=N_{2}^{1}$ denote a Klein bottle with one puncture $p$.

Lemma A.1. Let $c$ be a generic two-sided circle on $N$. Then $N \backslash c$ is connected and orientable.

Proof. Suppose that $N \backslash c$ has two components $M_{1}$ and $M_{2}$. Then both $M_{1}$ and $M_{2}$ have exactly one boundary component and one of them has a puncture. The Euler characteristics of $M_{1}$ and $M_{2}$ satisfy

$$
\chi\left(M_{1}\right)+\chi\left(M_{2}\right)=\chi(N)=-1 .
$$

Without loss of generality we can assume that $\chi\left(M_{1}\right) \geq \chi\left(M_{2}\right)$ and therefore $0 \leq \chi\left(M_{1}\right) \leq 1$. If $\chi\left(M_{1}\right)=1$ then $M_{1}$ is a disk, which is impossible since $c$ is generic. If $\chi\left(M_{1}\right)=0$ then $M_{1}$ is either a disk with a puncture or a Möbius strip. Both cases are impossible.

Since $\chi(N \backslash c)=-1$ and $N \backslash c$ has two boundary components and one puncture, if we glue a disk to each of the boundary components and remove 
the puncture, we obtain a surface of Euler characteristic 2, i.e. the sphere. Therefore $N \backslash c$ is orientable.

LemmA A.2. If $a$ and $b$ are generic two-sided circles on $N$, then there exists $h \in \mathcal{H}(N)$ such that $h(a)=b^{ \pm 1}$.

Proof. By the previous lemma, $N \backslash a$ and $N \backslash b$ are diffeomorphic as punctured surfaces. We can choose a diffeomorphism $h: N \backslash a \rightarrow N \backslash b$ which extends to $\widetilde{h}: N \rightarrow N$. Then $\widetilde{h}(a)=b^{ \pm 1}$.

Proposition A.3. There are exactly two isotopy classes of generic twosided circles on $N$.

Proof. By Lemma A.2, it is enough to prove that if we fix some generic two-sided circle on $N$ then $a \not a^{-1}$ and for any $h \in \mathcal{M}(N), h(a)$ is isotopic either to $a$ or to $a^{-1}$. To prove this, let us describe generators of $\mathcal{M}(N)$.

Following [8], we represent $N$ as the one-point compactification of a plane with two crosscaps and a puncture (see Figure 26). Let $\alpha, \beta, \gamma, a$ be closed curves indicated in Figure 26. In particular, $\beta$ and $\gamma$ are one-sided, while $\alpha$ and $a$ are two-sided. Define $v, w, y$ to be the puncture slides along $\beta$ and $\gamma$,

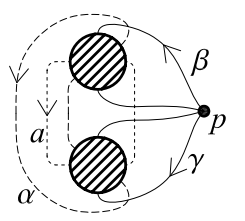

Fig. 26. Circles on a Klein bottle with puncture

and the crosscap slide along $\alpha$ respectively. Then by Theorem 4.9 of [8], $\mathcal{M}(N)$ is generated by $v, w, y$ and $t_{a}$.

It is straightforward to check that $a \neq a^{-1}, v(a) \simeq w(a) \simeq a^{-1}$ and $y(a) \simeq t_{a}(a)=a$.

COROLlaRY A.4. There is exactly one isotopy class of generic two-sided circles on a Klein bottle $\mathrm{N}_{2}$.

Consider another model of $N$, namely the one shown in Figure 27. Define

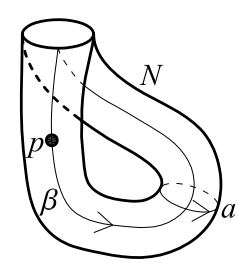

Fig. 27. Circles on a Klein bottle with puncture

$a$ and $\beta$ as shown in the figure, and let $v$ be the puncture slide along $\beta$. 
If we cut $N$ along $a$, we obtain a cylinder with a puncture. Reflection of this cylinder across the circle parallel to boundary components and passing through the puncture induces a diffeomorphism $\sigma \in \mathcal{H}(N)$ such that $\sigma(a)$ $=a^{-1}$.

THEOREM A.5. Let $N$ be a Klein bottle with a puncture and $v, \sigma$ as above. Then $\mathcal{M}(N)$ is the product $\left(\left\langle t_{a}\right\rangle \rtimes\langle v\rangle\right) \times\langle\sigma\rangle$ and is isomorphic to $\left(\mathbb{Z} \rtimes \mathbb{Z}_{2}\right) \times \mathbb{Z}_{2}$.

Proof. By Proposition A.3, if $h \in \mathcal{H}(N)$ is any diffeomorphism, then $h(a)$ is isotopic either to $a$ or to $a^{-1}$, so the subgroup $H<\mathcal{M}(N)$ consisting of maps which do not interchange the sides of $a$ is of index 2 in $\mathcal{M}(N)$. Moreover, $t_{a}, v \in H$ and $\sigma \in \mathcal{M}(N) \backslash H$.

All maps $h \in H$ such that $h(a)$ is isotopic to $a$ form a subgroup $K$ of index 2 in $H$, and $v \in H \backslash K$. If $k \in K$ is any diffeomorphism then we can assume that $k(a)=a$ and $k$ preserves the sides of $a$. If we cut $N$ open along $a$, we conclude from the mapping class group of the cylinder that $k=t_{a}^{n}$ for some $n \in \mathbb{Z}$. Therefore $H$ is generated by $v$ and $t_{a}$. Since $v^{2}$ is a twist about the boundary of a Möbius strip, $v$ is of order 2. Moreover, $v$ reverses the orientation of a regular neighbourhood of $a$, so $v t_{a} v^{-1}=t_{a}^{-1}$. Therefore

$$
H=\left\langle t_{a}\right\rangle \rtimes\langle v\rangle .
$$

Since $\sigma t_{a} \sigma^{-1}=t_{a}, \sigma v \sigma^{-1}=v^{-1}=v$, to complete the proof it is enough to show that $t_{a}$ is of infinite order. This can be shown by computing the induced homomorphism on homology.

Corollary A.6. Let $N$ be a Klein bottle with one puncture and $t_{a}, v, \sigma$ as above. Then the centre of $\mathcal{M}(N)$ is equal to the group of order 2 generated by $\sigma$. The centraliser $\mathcal{Z}$ of the twist subgroup is generated by $t_{a}$ and $\sigma$, and is isomorphic to $\mathbb{Z} \times \mathbb{Z}_{2}$.

A.2. Mapping class group of a Klein bottle with one boundary component. Now let $N=N_{2,1}$ denote the Klein bottle with one boundary component $b$. Observe that if $N^{\prime}$ is a Klein bottle with a puncture, then the inclusion $i: N \rightarrow N^{\prime}$ induces a homomorphism $i_{*}: \mathcal{M}(N) \rightarrow \mathcal{M}\left(N^{\prime}\right)$ which extends every $h \in \mathcal{M}(N)$ by the identity on $N^{\prime} \backslash N$ (see Figure 28; note that this time the shaded disk does not represent a crosscap but a disk). We claim that the kernel of $i_{*}$ is generated by the boundary twist $t_{b}$ on $N$. In fact, if $h \in \operatorname{ker} i_{*}$ then $h(a) \simeq a$ in $N^{\prime}$. By Proposition 3.5 of [10], we also have $h(a) \simeq a$ in $N$. Moreover, $h$ preserves the orientation of a neighbourhood of $a$, so it does not interchange the sides of $a$. Therefore $h$ is induced by a mapping of $\overline{N \backslash a}$, hence by the structure of the mapping class group of a pantalon of type III, $h=t_{a}^{\alpha} t_{b}^{\beta}$. Now $1=i_{*}(h)=t_{a}^{\alpha}$ and by Corollary 4.5 , $\alpha=0$. 


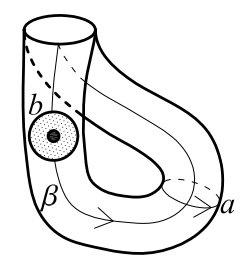

Fig. 28. Klein bottle with boundary as a subsurface of a Klein bottle with puncture

Since every diffeomorphism of $N$ fixes the boundary, the image of $i_{*}$ consists of elements of $\mathcal{M}\left(N^{\prime}\right)$ which preserve the local orientation around the puncture on $N^{\prime}$. All such elements form a subgroup $\mathcal{M}^{+}\left(N^{\prime}\right)$ of index 2 , which is generated by $t_{a}$ and $\sigma v$. Observe that we can use the same definitions as for the maps $t_{a}, \sigma$, and $v$ to define diffeomorphisms $t_{a}, \widetilde{\sigma}, \widetilde{v}: N \rightarrow N$ such that $i_{*}\left(t_{a}\right)=t_{a}, i_{*}(\widetilde{\sigma})=\sigma, i_{*}(\widetilde{v})=v$. The problem is that $\widetilde{\sigma}$ and $\widetilde{v}$ do not fix the boundary of $N$. However, if we define

$$
\widetilde{\sigma v}=\widetilde{\sigma} \widetilde{v} \bar{t}_{b}
$$

where $\bar{t}_{b}$ is a half twist about the boundary circle $b$, then $\widetilde{\sigma v}: N \rightarrow N$ fixes the boundary and $i_{*}(\widetilde{\sigma v})=\sigma v$. Now from the exact sequence

$$
1 \rightarrow\left\langle t_{b}\right\rangle \rightarrow \mathcal{M}(N) \stackrel{i_{*}}{\rightarrow} \mathcal{M}^{+}\left(N^{\prime}\right) \rightarrow 1
$$

and easily verifiable relations

$$
\widetilde{\sigma v}^{2}=t_{b}, \quad \widetilde{\sigma v} t_{a} \widetilde{\sigma v}^{-1}=t_{a}^{-1}
$$

we obtain the following theorem:

THEOREM A.7. Let $N$ be a Klein bottle with one boundary component and $t_{a}, \widetilde{\sigma v}$ as above. Then the mapping class group of $N$ is the semidirect product $\left\langle t_{a}\right\rangle \rtimes\langle\widetilde{\sigma v}\rangle$ and is isomorphic to $\mathbb{Z} \rtimes \mathbb{Z}$.

Corollary A.8. Let $N$ be a Klein bottle with one boundary component and $t_{a}, \widetilde{\sigma v}, t_{b}=\widetilde{\sigma v}^{2}$ as above. Then the centre of $\mathcal{M}(N)$ is the cyclic group generated by $t_{b}$. The centraliser $\mathcal{Z}$ of the twist subgroup is generated by $t_{a}$ and $t_{b}$, and is isomorphic to $\mathbb{Z} \times \mathbb{Z}$.

Acknowledgements. The author wishes to thank the referee for his/her helpful suggestions.

\section{References}

[1] J. S. Birman and D. R. J. Chillingworth, On the homeotopy group of a non-orientable surface, Proc. Cambridge Philos. Soc. 71 (1972), 437-448; erratum, ibid. 136 (2004), 441.

[2] D. R. J. Chillingworth, A finite set of generators for the homeotopy group of a non-orientable surface, ibid. 65 (1969), 409-430. 
[3] D. B. A. Epstein, Curves on 2-manifolds and isotopies, Acta Math. 115 (1966), 83-107.

[4] A. Fathi, F. Laudenbach, and V. Poénaru (eds.), Travaux de Thurston sur les surfaces, Séminaire Orsay, Astérisque 66-67 (1979).

[5] N. V. Ivanov, Mapping class groups, in: R. J. Daverman and R. B. Sher (eds.), Handbook of Geometric Topology, Elsevier, 2002, Chapter 12, 523-633.

[6] N. V. Ivanov and J. D. McCarthy, On injective homomorphisms between Teichmüller modular groups I, Invent. Math. 135 (1999), 425-486.

[7] M. Korkmaz, First homology group of mapping class groups of nonorientable surfaces, Math. Proc. Cambridge Philos. Soc. 123 (1998), 487-499.

[8] -, Mapping class groups of nonorientable surfaces, Geom. Dedicata 89 (2002), 109133.

[9] W. B. R. Lickorish, Homeomorphisms of non-orientable two-manifolds, Math. Proc. Cambridge Philos. Soc. 59 (1963), 307-317.

[10] L. Paris and D. Rolfsen, Geometric subgroups of mapping class groups, J. Reine Angew. Math. 521 (2000), 47-83.

[11] B. Szepietowski, Mapping class group of a non-orientable surface and moduli space of Klein surfaces, C. R. Acad. Sci. Paris Sér. I 335 (2002), 1053-1056.

[12] - Involutions in mapping class groups of nonorientable surfaces, Collect. Math. 55 (2004), 253-260.

Institute of Mathematics

University of Gdańsk

Wita Stwosza 57

80-952 Gdańsk, Poland

E-mail: trojkat@math.univ.gda.pl

Received 25 October 2004;

in revised form 8 November 2005 